\title{
On the storm-time evolution of relativistic electron phase space density in Earth's outer radiation belt
}

\author{
D. L. Turner, ${ }^{1}$ V. Angelopoulos, ${ }^{1}$ W. Li, ${ }^{2}$ M. D. Hartinger ${ }^{3}$ M. Usanova, ${ }^{4}$ I. R. Mann, ${ }^{4}$ \\ J. Bortnik, ${ }^{2,5}$ and Y. Shprits ${ }^{1,6,7}$ \\ Received 23 October 2012; revised 17 January 2013; accepted 25 January 2013; published 24 May 2013.
}

[1] We report on internal, magnetospheric processes related to markedly different storm-time responses of phase space density (PSD) in invariant coordinates corresponding to equatorially mirroring, relativistic electrons in Earth's outer radiation belt. Two storms are studied in detail, selected from a database of 53 events (Dst ${ }_{\min }<-40 \mathrm{nT}$ ) during the THEMIS era thus far (December 2007-August 2012). These storms are well covered by a number of in situ THEMIS spacecraft and complemented by additional ground-based and in situ observatories, and they epitomize the divergent behaviors that the outer radiation belt electrons can exhibit during active periods, even during otherwise similar Dst and auroral electrojet (AE) profiles. From our statistical results with the full database, the changes in the radial profile peak in PSD reveal notably consistent behavior with prior studies: $58 \%$ of geomagnetic storms resulted in PSD peak enhancements, $17 \%$ resulted in PSD peak depletions, and $25 \%$ resulted in no significant change in the PSD peak after the storm. For the two case studies, we examined the PSD at multiple equatorial locations (using THEMIS), trapped and precipitating fluxes from low-Earth orbit (using POES), and chorus, hiss, EMIC, and ULF waves (using THEMIS spacecraft, ground observatories, and the GOES spacecraft). We show that (1) peaks in PSD were collocated with observed chorus waves outside of the plasmapause during the most active periods of the PSD-enhancing storm but not during the PSD-depleting storm, providing evidence for the importance of local acceleration by wave-particle interactions with chorus; (2) outer belt dropouts occurred following solar wind pressure enhancements during both storms and were consistent with losses from magnetopause shadowing and subsequent outward radial transport; during the PSD-enhancing storm, this revealed how the outer belt can replenish itself seemingly independently of the remnant of the pre-existing belt leftover after a dropout, which in this case resulted in a double-peaked outer belt distribution; (3) slow decay in PSD was associated with corresponding locations in $\mathrm{L}^{*}$ and enhanced wave amplitudes of plasmaspheric hiss; (4) precipitation loss associated with wave-particle interactions with hiss and EMIC waves appeared to be significantly more important during the PSD-depleting storm than the PSD-enhancing storm; and (5) PSD transport during the recovery phase of both storms and throughout the PSD-enhancing storm was consistent with ULF-wave-driven radial diffusion away from maxima in PSD; this indicates the importance of ULF waves in redistributing outer belt PSD after local acceleration occurs. We conclude that these source, transport, and loss processes, individually well characterized by previous studies, do indeed appear to act in concert, leading to predominance of local acceleration in one case and loss in another. These processes can therefore conspire toward optimal source or loss of outer belt electrons under suitable external drivers, and the conditions resulting in wave growth for these acceleration and loss mechanisms are therefore an important area of future research.

\footnotetext{
${ }^{1}$ Department of Earth and Space Sciences, University of California, Los Angeles, California, USA.

${ }^{2}$ Department of Atmospheric and Oceanic Sciences, University of California, Los Angeles, California, USA.

${ }^{3}$ Department of Atmospheric, Oceanic, and Space Sciences, University of Michigan, Ann Arbor, Michigan, USA.

${ }^{4}$ Department of Physics, University of Alberta, Edmonton, Alberta, AB, Canada.

${ }^{5}$ Visiting scholar at New Jersey Institute of Technology, Newark, New Jersey, USA.

${ }^{6}$ Skolkovo Institute of Science and Technology, Moscow, Russia.

${ }^{7}$ Visiting professor at Massachusetts Institute of Technology, Cambridge, Massachusetts, USA.
} 
Citation: Turner, D. L., V. Angelopoulos, W. Li, M. D. Hartinger, M. Usanova, I. R. Mann, J. Bortnik, and Y. Shprits (2013), On the storm-time evolution of relativistic electron phase space density in Earth's outer radiation belt, J. Geophys. Res. Space Physics, 118, 2196-2212, doi:10.1002/jgra.50151.

\section{Introduction}

[2] Earth's outer radiation belt consists of a highly variable population of trapped relativistic electrons in near-Earth space, which can pose a threat to manmade systems [Baker, 2002]. Internal magnetospheric processes, which are ultimately driven by energy provided via the solar wind [Li et al., 1997], are responsible for the various acceleration (i.e., source), loss, and transport mechanisms that drive the orders-of-magnitude variations observed in the outer belt population. These variations occur over a broad range of time scales, from minutes to years [see reviews by Friedel et al., 2002; Millan and Thorne, 2007; Shprits et al., 2008a, 2008b; Turner et al., 2012c]. The outer belt population is particularly dynamic during geomagnetic storms, when magnetospheric activity levels peak [e.g., Baker et al., 1998; Bortnik et al., 2006]. Reeves et al. [2003] conducted a study of the outer belt electron flux response, as observed by the LANL-GEO and POLAR spacecraft, to 276 storms characterized by a minimum Dst index level of less than $-50 \mathrm{nT}$. They found that $53 \%$ of storms resulted in an enhancement in the peak flux level of relativistic electrons at geosynchronous orbit $(>2$ times increase from pre- to post-storm), $28 \%$ resulted in little change in the peak flux, and the remaining $19 \%$ resulted in a decrease of the peak flux level ( $>2$ times decrease from pre- to post-storm). Their conclusion was that the statistics were evidence of a "delicate and complicated balance" [Reeves et al., 2003, p. 36-1] between competing source, loss, and transport mechanisms.

[3] Several mechanisms have been identified that can theoretically result in the acceleration of electrons to relativistic energies in Earth's outer radiation belt, and the majority of these theories can be categorized into three groups: external sources, internal sources, and recirculation models. The framework of most of these theories relies heavily on quasi-linear diffusion theory in one or more dimensions (using phase space density in 3-D invariant coordinates by averaging over the phases of the three characteristic periodic motions) [Schulz and Lanzerotti, 1974]. External sources require a reservoir of electron phase space density (PSD) in Earth's magnetotail, and electrons from this reservoir can be introduced to the inner magnetosphere via transport mechanisms that break the third adiabatic (i.e., drift) invariant, L* [Roederer, 1970]. With a source at the outer boundary, inward transport and energization is enabled by ULF waves in the Pc5 frequency range, which break the third adiabatic invariant and provide the random displacements in L* necessary for diffusion [e.g., Hudson et al., 2000; Elkington et al., 2003; Mann et al., 2004]. Since the first and second invariants ( $\mathrm{Mu}$ and $\mathrm{K}$, respectively) are conserved, electrons become energized in the process of inward transport to more dipolar regions with higher magnetic field strengths and shorter field line lengths. Sudden injections from a PSD source at high $\mathrm{L}^{*}$ by either sub-storm dipolarization fronts [e.g., Ingraham et al., 2001] or interplanetary shocks [e.g., Li et al., 1998] also fall into this external source category.
[4] Alternatively, wave-particle interactions (WPI) between a seed population of electrons at several tens to a few hundreds of kiloelectronvolt and naturally occurring plasma waves within the outer belt region can result in net acceleration of a percentage of those electrons to relativistic energies. These WPI mechanisms fall into the internal source category of radiation belt acceleration. Waves that have been identified as potentially important to this are whistler-mode chorus [e.g., Summers et al., 1998; Horne and Thorne, 1998; Meredith et al., 2002; 2003; Horne et al., 2005; Li et al., 2007] and magnetosonic waves (i.e., equatorial noise) [Horne et al., 2007]. Finally, recirculation models represent scenarios in which electrons are energized repeatedly by circuitous trajectories through an acceleration region. These models require non-adiabatic variations in equatorial pitch angle and transport across drift shells. This can occur at high latitudes after acceleration closer to the equator and subsequent pitch-angle scattering by WPI [Fujimoto and Nishida, 1990]. It can also occur by Mu-conserving random walks in bifurcated drift orbits (i.e., Shabansky orbits, after Shabansky [1971]), which break electrons' second and third invariants at higher $\mathrm{L}^{*}$ in the dayside magnetosphere due to the topology of the compressed magnetic field [Ukhorskiy et al., 2011].

[5] In addition to acceleration, WPI can also result in a loss of relativistic electrons from the outer belt by way of pitch-angle scattering into the atmospheric loss cones [e.g., Shprits et al., 2006a]. Waves that have been identified as important in such loss processes include plasmaspheric hiss [e.g., Lyons et al., 1972; Li et al., 2007; Meredith et al., 2009], whistler-mode chorus [e.g., Horne and Thorne, 2003; O'Brien et al., 2004; Bortnik and Thorne, 2007], whistler waves originating from lightning and ground-based VLF transmitters [e.g., Abel and Thorne, 1998a; 1998b], and electromagnetic ion cyclotron (EMIC) waves [e.g., Summers and Thorne, 2003; Albert, 2003]. Precipitating relativistic electrons are observable by certain spacecraft in low-Earth orbits (LEO) [e.g., O'Brien et al., 2004; Meredith et al., 2011] and can also be observed indirectly via highaltitude balloons that detect Bremsstrahlung X-rays produced by collisions between relativistic electrons and neutral particles in Earth's atmosphere [e.g., Millan et al., 2002]. Precipitation events appear as spatially confined, longer-duration precipitation bands of enhanced flux in the loss cone [e.g., Millan et al., 2010] or as intense, short-lived (i.e., millisecond durations) micro-bursts [e.g., O'Brien et al., 2004]. Previous studies [e.g., Thorne et al., 2005; Selesnick, 2006; Tu et al., 2010; Ukhorskiy et al., 2010] have also concluded that during the main phase of some storms, losses of greater than $\sim 0.5 \mathrm{MeV}$ electrons to the atmosphere due to wave-particle interactions can be effective over timescales ranging from hours to around 1 day and might explain losses during flux "dropouts."

[6] Trapped electrons can also be lost to the outer boundary of the system through the dayside magnetopause. This happens due to sudden inward motion of the magnetopause in response to increased solar wind dynamic pressure and 
is known as magnetopause shadowing [e.g., Ukhorskiy et al., 2006]. Recent studies concluded that a combination of magnetopause shadowing and subsequent enhanced outward radial transport (e.g., by enhanced ULF wave activity) can account for observations of sudden dropouts of electrons throughout the outer radiation belt [e.g., Shprits et al., 2006b; Loto'aniu et al., 2010; Turner et al., 2012a]. Such a loss model is further supported by the results of Shprits et al. [2012], who conducted a statistical study of reanalyzed electron PSD throughout the outer belt and found $88 \%$ of dropouts were associated with increases in solar wind dynamic pressure [see also Kellerman and Shprits, 2012].

[7] All source, loss, and transport mechanisms discussed here are dependent on the solar wind and its resultant geomagnetic activity; the effects of these mechanisms on the outer belt electron population are believed to be more intense during specific active conditions that can occur during geomagnetic storms. However, validating these theories with observational evidence is challenging for several reasons. First, the outer belt system is enormous, wrapping entirely around the planet at radial distances between $\sim 3$ and $\sim 7$ Earth radii $\left(R_{E}\right)$ in the equatorial plane and stretching along magnetic field lines from the equatorial plane to Earth's atmosphere in both the northern and southern hemispheres. At any given time, there are only a few spacecraft making in situ observations of this vast system. Thus, multi-point studies are critical for developing a more complete picture of the entire system. Next, relativistic electron measurements over a finite range of energies and local pitch angles by themselves do not provide an accurate picture of source, loss, and transport mechanisms, since they naturally include adiabatic transport effects [e.g., Kim and Chan, 1997; Tu and Li, 2011] and drift shell splitting [e.g., Selesnick and Blake, 2002]. These ambiguities are removed by converting flux measurements to PSD as a function of the three adiabatic invariants [e.g., Green and Kivelson, 2004]. Recent studies of outer belt electron PSD in invariant coordinates have revealed evidence of peaked PSD distributions in L* [e.g., Green and Kivelson, 2004; Chen et al., 2007; Turner et al., 2012b], local acceleration within the outer belt [e.g., Miyoshi et al., 2003; Chen et al., 2007; Shprits et al., 2007; Turner et al., 2010; Schiller et al., 2012], and Mu-dependent $\mathrm{L}^{*}$ distributions of PSD [e.g., Turner and Li, 2008; Turner et al., 2012b]. Finally, with the exception of those using CRRES data, most past studies have been unable to adequately discern the evolution of equatorial fluxes throughout the entire outer belt because they have relied on high-inclination spacecraft that often miss the (typically higher) fluxes of equatorially mirroring electrons and their evolution.

[8] Here, we examine the PSD in invariant coordinates methodology to compare and contrast the PSD evolution of equatorially mirroring, relativistic electrons throughout Earth's outer radiation belt using the near-equatorial THEMIS spacecraft, the orbits from which (plus the GOES spacecraft) are shown in Figure 1 for the two events detailed in this study. The recent increase in solar activity and the unique, near-equatorial, and high-eccentricity orbits of THEMIS, which provide radial distributions of PSD throughout the outer radiation belt, have enabled this study. To examine the details of spatio-temporal evolution of the PSD, we focus on two different geomagnetic storms: one storm resulting in
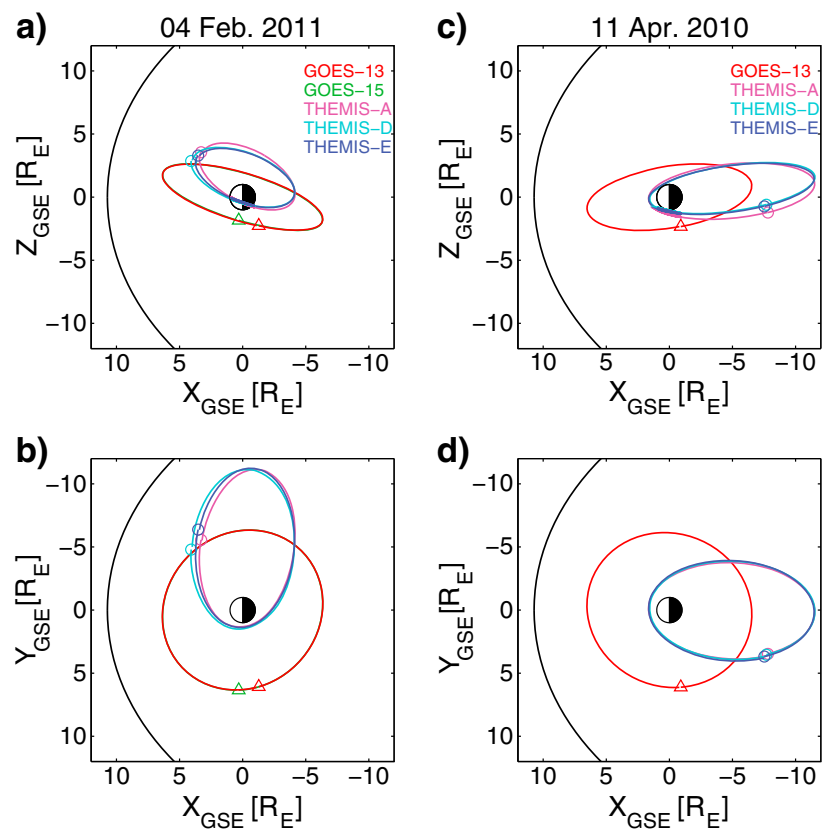

Figure 1. Spacecraft orbits during the two events discussed here. ( $a$ and $b$ ) show the spacecraft locations at 00:00 UT on 04 February 2011 and the orbit tracks for $24 \mathrm{~h}$ after that in the GSE XZ and XY planes, respectively. (c and d) show the same for 23:59 UT on 11 April 2010 and the orbit tracks for $24 \mathrm{~h}$ after that in the same format. Note that orbital motion for all spacecraft shown is in the right-handed sense with respect to the XY plane. GOES spacecraft at GEO are indicated with triangle symbols, while THEMIS are indicated with circles. Each spacecraft and the respective orbit is shown in a corresponding color, as indicated in the legends in Figures 1a and $1 \mathrm{c}$.

an overall enhancement and the other resulting in a prolonged depletion of the PSD throughout the outer belt. We also revisit the results of Reeves et al. [2003] using PSD for fixed adiabatic invariants instead of flux as a function of energy and local pitch angle.

\section{Data}

[9] For this study, we incorporated data from multiple missions. Supplementing data from NASA's THEMIS spacecraft and ground-based observatories (GBOs) [Angelopoulos, 2008], we employed measurements from NOAA's GOES spacecraft at geosynchronous orbit (GEO) and NOAA's POES spacecraft in low-Earth orbit (LEO). We used relativistic electron flux measurements provided by the THEMIS-SST instruments and the POES MEPED instruments [Evans and Greer, 2004]. Due to early mission commissioning, we used data after December 2007 only. The SSTs provide full distributions of energetic electrons in 12 channels ranging in energy from $\sim 26 \mathrm{keV}$ to $>1 \mathrm{MeV}$. Here we only used the four highest energy channels (ranging from $\sim 400 \mathrm{keV}$ to several $\mathrm{MeV}$ ) that employ coincidence logic and were thus unaffected by energetic protons in the inner magnetosphere, which can otherwise contaminate energetic electron measurements. Fluxes from these four channels were converted to phase space density for fixed first and second adiabatic invariant coordinates of $\mathrm{Mu}=1000 \mathrm{MeV} / \mathrm{G}$ 
(corresponding to $\sim 650 \mathrm{keV}$ electrons near GEO and $\sim 1.2 \mathrm{MeV}$ electrons in the heart of the belt at $\mathrm{L}^{*} \sim 5$ ) and $\mathrm{K} \sim 0 \quad \mathrm{G}^{1 / 2} \mathrm{R}_{\mathrm{E}}$ (near equatorially mirroring electrons, with equatorial pitch angles in the range $90+/-15^{\circ}$ ) at all $\mathrm{L}^{*}$ throughout the outer belt using the method described in Turner et al. [2012b]. The POES MEPED fluxes shown in this paper are from the $>6.9 \mathrm{MeV}$ proton channel, which responds to $>1 \mathrm{MeV}$ electrons throughout the outer radiation belt [Yando et al., 2011]. The $>30 \mathrm{keV},>100 \mathrm{keV}$, and $>300 \mathrm{keV}$ electron channels were also examined, though we do not discuss the results from those here. All of the MEPED data were decontaminated using the Lam et al. [2010] method.

[10] Wave data were compiled from THEMIS and GOES spacecraft and a network of ground magnetometers (GMAGs). Wave amplitudes of chorus and hiss were obtained from filter bank data (FBK) comprised of the wave magnetic field measured by THEMIS search coil magnetometers (SCM) [Roux et al., 2008]. Total plasma density inferred from the spacecraft potential was used to determine if the waves were observed in the plasmasphere-like or trough-like region following the method of Li et al. [2010]. Since the frequency range of plasmaspheric hiss is from $\sim 100 \mathrm{~Hz}$ to a few $\mathrm{kHz}$ and it is generally confined within the plasmasphere [e.g., Thorne et al., 1973], its amplitude in each time stamp was calculated based on the root mean square (RMS) of wave amplitude in the top three frequency bands $(80.2 \mathrm{~Hz}-4 \mathrm{kHz})$ in the FBK data, when the spacecraft was located inside the plasmasphere. Chorus waves are typically observed in the low-density region outside the plasmapause, in the frequency range of $0.1-0.8$ fce, where fce is the equatorial electron cyclotron frequency. The fce was calculated based on the locally measured magnetic field intensity on the THEMIS fluxgate magnetometers (FGM) [Auster et al., 2008]. When the spacecraft were outside the plasmapause, the calculated fce was used at each time stamp to select the appropriate frequency bands to calculate the amplitude of chorus waves, using the method described in detail in Li et al. [2009].

[11] EMIC waves at GEO were identified using GOES-13 and -15 fluxgate magnetometers that can detect magnetic field variations in the frequency range from $\mathrm{DC}$ to $1 \mathrm{~Hz}$ [see, e.g., Singer et al., 1996]. For ground-based analysis, we utilized magnetic field data from multiple search coil magnetometers around the globe. In particular, we used induction coil magnetometer data from selected stations in the Canadian Array for Real-time Investigations of Magnetic Activity (CARISMA) [www.carisma.ca; Mann et al., 2008] between $\mathrm{L}=4.06-6.81$, the Sodankylä Geophysical Observatory in Finland (www.sgo.fi/Data/Pulsation) between $\mathrm{L}=3.3-5.9$, and Halley Bay in Antarctica (space.augsburg. edu/searchcoil) at $\mathrm{L}=4.3$. EMIC waves can be ducted in the ionosphere and can propagate a long distance from the source field line. Hence, for CARISMA and Sodankylä stations, we selected intervals where the most intense wave activity (with respect to the neighboring magnetometers) was observed. If the wave intensity did not vary much from station to station, we assumed that the wave source spanned a range of L-shells in the magnetosphere.

[12] GOES magnetometer data sampled every $512 \mathrm{~ms}$ were obtained from the National Geophysical Data Center (http://www.ngdc.noaa.gov/stp/satellite/goes/index.html). Flagged data were removed, small gaps were interpolated, and the data were converted to $3 \mathrm{~s}$ time resolution. We performed a 512 point Fast Fourier Transform on the perturbation fields $(>1 \mathrm{mHz})$ to compute the compressional power spectral density $\left(\mathrm{P}_{\text {btotal }}\right)$ and transverse power spectral density $\left(\mathrm{P}_{\mathrm{btrans}}=\mathrm{P}_{\mathrm{bz}}+\mathrm{P}_{\mathrm{by}}+\mathrm{P}_{\mathrm{bx}}-\mathrm{P}_{\mathrm{btotal}}\right.$, where $\mathrm{xyz}$ is in local spacecraft coordinates); see the Figure 7 caption for additional details. We finally computed the GOES Pc5 power spectral density by averaging the power spectral densities over the 2-7 $\mathrm{mHz}$ frequency band; we normalized the GOES Pc5 power spectral density to the maximum value found during both storm intervals.

[13] Solar wind and geomagnetic indices are from the OMNI dataset available at NASA's CDAWeb (http://cdaweb. gsfc.nasa.gov/). Hourly OMNI data were utilized in this study, though higher resolution OMNI data $(1 \mathrm{~min})$ were used as input for magnetic field modeling for $\mathrm{K}$ and $\mathrm{L}^{*}$ calculations and for the ULF wave analysis. The Tsyganenko and Sitnov [2005] field model was used with the SpacePy/IRBEM toolsets [see http://spacepy.lanl.gov/; http://craterre.onecert. $\mathrm{fr} / /$ prbem/home.html] for calculating $\mathrm{K}$ and $\mathrm{L}^{*}$ for the THEMIS-SST data. From the OMNI Dst index data, we identified 53 storms between December 2007 and August 2012 in which the minimum Dst was less than $-40 \mathrm{nT}$ during the main phase and the storm sudden commencement (SSC) reached Dst $>0$ [for details on storm phases, see Kamide et al., 1998]. For these storms, we calculated the electron PSD for $\mathrm{Mu}=1000 \mathrm{MeV} / \mathrm{G}$ and $\mathrm{K} \sim 0 \mathrm{G}^{1 / 2} \mathrm{R}_{\mathrm{E}}$ and classified storms as having resulted in an enhancement, a depletion, or no change in the peak in PSD throughout the belt as follows. Following Reeves et al. [2003], we classified a storm as resulting in enhancement (depletion) of relativistic electrons when the maximum peak in PSD throughout the outer belt, regardless of its location in $\mathrm{L}^{*}$, increased (decreased) by at least a factor of 2 from the 2 days prior to the main phase to 2 days after it. Storms classified as resulting in "no change in PSD" were those that did not satisfy either of the enhancement or depletion criteria. Of the 53 storms with main phase minimum Dst $<-40 \mathrm{nT}$, $58 \%$ result in an enhancement in the peak PSD level, $17 \%$ result in a depletion in the peak PSD level, and $25 \%$ result in no significant change. These statistics are notably similar to those of Reeves et al. [2003], and in the next sections, we present a detailed analysis of two of these storms, one an enhancement and one a depletion event.

\section{Observations}

\subsection{Solar Wind and Geomagnetic Activity}

[14] Solar wind and geomagnetic indices for the two storms that we analyzed in detail are shown in Figure 2, along with a timeline of key features during each storm period. The 04 February 2011 storm resulted in a net enhancement of PSD throughout the outer belt. In the days leading up to the SSC, a high-speed stream in the solar wind occurred from 01 to 04 February, with the maximum speed reaching over $500 \mathrm{~km} / \mathrm{s}$ late in the day on 01 February. A stream-interface region (SIR), visible as the strong dynamic pressure enhancement at the end of the day on 31 January, preceded this high-speed stream. This SIR and high-speed stream did not result in a geomagnetic storm, as is evident in the Dst index, but they did correspond to some sub-storm activity apparent in the AL index, particularly around the 

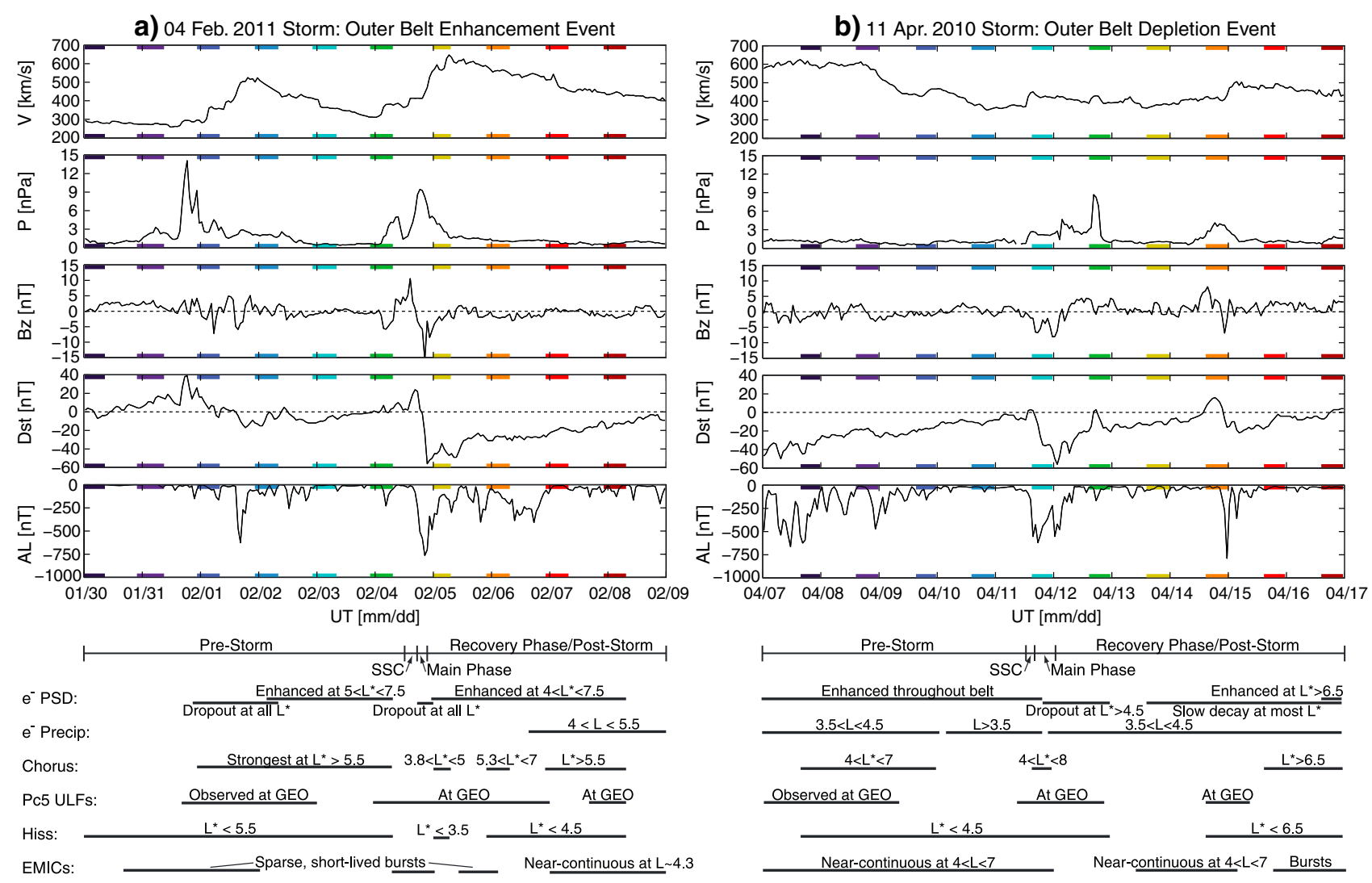

Figure 2. Solar wind and geomagnetic indices during both events. (a) Solar wind speed (V), dynamic pressure $(\mathrm{P})$, and the GSM Z-component of the IMF vector $(\mathrm{Bz})$, with the Dst and AL indices for the 10 day period from 30 January-08 February 2011. The colored bars on the $X$ axis correspond to the time periods in which the THEMIS spacecraft were in the outer radiation belt, corresponding to the same colors in the distributions shown in Figure 3 ( $a$ and b). (b) The same quantities shown in (a) but for the 10 day period from 07 to 16 April 2010. The colored bars again represent periods when THEMIS was in the outer belt and correspond to the same colors in the distributions shown in Figure 3 (c and d) and the wave data shown in Figures 5, 6, and 8. Below these plots, timelines corresponding to the periods shown on the $x$ axes identify key features for each storm, including (i) the storm phases [Kamide et al., 1998], (ii) electron phase space density dynamics (e-PSD), (iii) electron precipitation into the atmosphere (e-Precip.), (iv) chorus wave activity, (v) Pc5 ULF wave activity, (vi) hiss wave activity, and (vii) EMIC wave activity.

middle of the day on 01 February. The storm examined here was the result of a second high-speed stream preceded by a SIR starting at the beginning of the day on 04 February. The storm started around 12:00 UT on 04 February, corresponding to the second and stronger of two distinct solar wind dynamic pressure enhancements. The main phase of the storm followed, reaching a minimum Dst level of greater than $-60 \mathrm{nT}$ before the end of the day. As is typical of SIR driven storms [e.g., Morley et al., 2010], the recovery phase was prolonged for this event, lasting until 08 February. During this recovery phase, the IMF was primarily southward, and there was strong sub-storm activity, particularly from the storm main phase until the end of the day on 06 February.

[15] The storm on 11 April 2010 resulted in a prolonged (i.e., several-day) depletion of PSD throughout the outer belt. This storm occurred at the end of the recovery phase of a much larger storm that started on 05 April due to a coronal mass ejection (CME) impact. The high-speed solar wind after this CME is evident on the first few days shown in Figure 2b. The recovery phase of the 05 April storm revealed enhanced sub-storm activity, evident in the AL index data. In the early afternoon on 11 April, the solar wind speed and pressure exhibited a small, sharp jump in their magnitudes. These are possibly associated with a glancing impact from a smaller CME ejected from the same active area, which is consistent with evidence of a magnetic cloud in the IMF between $\sim 12: 00$ UT on 11 April and 19:00 UT on 12 April during which the pressure remained higher than average and the IMF Bz rotated southward to northward. Additionally, there was evidence of a reverse shock [Gonzalez et al., 1999] at the trailing edge of this magnetic cloud around 17:00-19:00 UT on 12 April. The SSC started at the impact of the magnetic cloud after noon on 11 April. The main phase reached a minimum Dst of greater than $-60 \mathrm{nT}$ at $\sim 02: 00 \mathrm{UT}$ on 12 April, and around this main phase, there was less than $24 \mathrm{~h}$ of enhanced sub-storm activity evidenced by the AL index. The recovery phase lasted until 14 April, but it was interrupted by the pressure enhancement associated with the reverse shock on 12 April. The solar wind speed remained around its average level ( $\sim 425 \mathrm{~km} / \mathrm{s})$ throughout the storm. At the end of the day on 14 April, another solar wind dynamic pressure enhancement occurred with a slight increase in the solar wind speed up to $\sim 500 \mathrm{~km} / \mathrm{s}$. This was 
accompanied by a brief period of strong southward IMF and a strong sub-storm starting around 23:00 UT on 14 April.

\subsection{Evolution of Phase Space Density Distributions in $L^{*}$}

[16] Electron PSDs for $\mathrm{Mu}=1000 \mathrm{MeV} / \mathrm{G}$ and $\mathrm{K}$ $0.025 \mathrm{G}^{1 / 2} \mathrm{R}_{\mathrm{E}}$ from both events are shown in Figure 3 . Note that for the February 2011 storm, THEMIS-A (TH-A) was consistently closer to the magnetic equator than TH-D and TH-E; thus, the $\mathrm{K}$ corresponding to the TH-A PSDs is consistently closer to $0 \mathrm{G}^{1 / 2} \mathrm{R}_{\mathrm{E}}$ (in fact, it is consistently $<0.01 \mathrm{G}^{1 / 2} \mathrm{R}_{\mathrm{E}}$, corresponding to electrons with equatorial pitch angles within $90 \pm 10^{\circ}$ ). During this storm, the three THEMIS spacecraft were inbound in the late morning MLT sector, between $\sim 8$ and 11 MLT. They were outbound in the early morning MLT sector, between $\sim 1$ and 4 MLT. For the April 2010 storm, again TH-A was closest to the magnetic equator, but all of the spacecraft were closer to each other than during the February 2011 event; the K corresponding to all of them is less than $0.01 \mathrm{G}^{1 / 2} \mathrm{R}_{\mathrm{E}}$. At this time, the three THEMIS spacecraft were inbound in the early morning MLT sector, between $\sim 3$ and 6 MLT. They were outbound in the evening MLT sector, between $\sim 19$ and 22 MLT. For both storms, there was approximately $3 \mathrm{~h}$ near perigee between inbound and outbound orbital trajectories in the outer belt. Also during both storm periods, TH-D and TH-E had conjunctions in phase space coordinates, allowing their PSDs to be inter-calibrated [e.g., Ni et al., 2011] for direct comparison. Finally, note that the bounds in $\mathrm{L}^{*}$ of the PSD distributions shown in Figure 3 are the result of either $\mathrm{L}^{*}$ being undefined by the model (most often corresponding to the outer bound on the plot) or the energy required to conserve $\mathrm{Mu}$ extending outside of the range of the four SST energy channels used to calculate PSD (corresponding to all of the inner bounds and several of the outer).

[17] During the course of the February 2011 storm, the outer belt started out with a peak in PSD at $4.5<\mathrm{L}^{*}<5$.
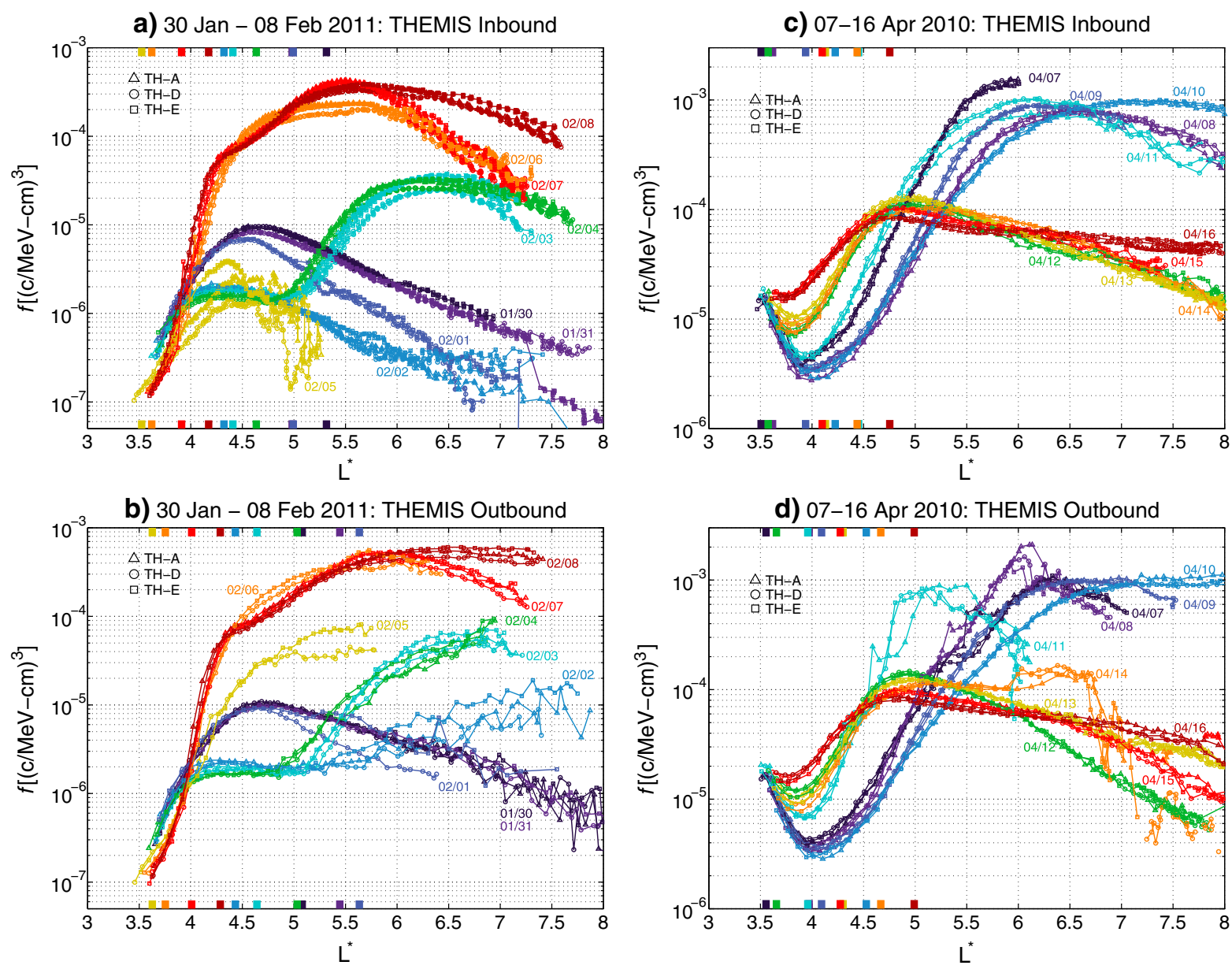

Figure 3. Phase space density $\mathrm{L}^{*}$ distributions for $\mathrm{Mu}=1000 \mathrm{MeV} / \mathrm{G}$ and $\mathrm{K}<0.025 \mathrm{G}^{1 / 2} \mathrm{R}_{\mathrm{E}}$ during the 10 day periods surrounding two geomagnetic storms. For the February 2011 storm, distributions from (a) inbound portions of the THEMIS orbits and (b) outbound trajectories are shown with symbols corresponding to the three THEMIS spacecraft (triangles: TH-A; circles: TH-D; squares: TH-E) and colors (also labeled with date in month/day format) corresponding to the times shown in Figure 2a. The plasmapause locations, calculated using the O'Brien and Moldwin [2003] model using Dst, are indicated for each day with the corresponding colored bars on the $X$ axis. Note that the inbound trajectories occurred before the outbound trajectories of the same color. (c and d) show the distributions from the April 2010 storm in the same format but with colors corresponding to those shown in Figure $2 \mathrm{~b}$. 
This exhibited slow decay over the first 2 days of this period. Then on the inbound trajectories at the beginning of 01 February, right after the pressure enhancement in the SIR, the PSD distribution started showing enhanced losses, which became more pronounced with increasing $L^{*}$. Note that the outbound orbit on 01 February revealed slightly higher PSD levels at higher $\mathrm{L}^{*} \mathrm{~s}$ than the inbound trajectory, indicating a local time effect of the PSD radial profiles (recall that all three THEMIS spacecraft were in the late morning MLT sector during the inbound trajectories and early morning MLT sector during the outbound trajectories of their orbits). We propose a few different possible explanations for this difference. Part of the difference may be due to the slight differences in K (i.e., THEMIS were slightly closer to or farther from the magnetic equator) between the inbound and outbound trajectories. Another reason may possibly be that part of the difference results from differences in $\mathrm{L}^{*}$ due to magnetic field model inaccuracies, which may be stronger at higher radial distances. Finally, part of the difference may also result from Shabansky orbits becoming increasingly more important in describing particle drift paths, due to the enhanced solar wind dynamic pressure. Such drift orbits would result in reducing the flux of equatorially mirroring electrons on the dayside between around 07:00 and 17:00 MLT [e.g., Ukhorskiy et al., 2011]. Also, from the outbound trajectories, it appears in Figure $3 \mathrm{~b}$ as though the PSD was highest at the outer trapping boundary (corresponding to the maximum $\mathrm{L}^{*}$ defined by the global field model), but when PSD for fixed first and second invariants was examined at higher radial distances, they revealed that all of these distributions were indeed peaked beyond the model boundary deeper into the tail. Continuing with the evolution throughout the storm, the result of the dropout starting on 01 February was the distribution seen on 02 February, namely a distribution with a peak in PSD at $L^{*} \sim 4.5$ at only $\sim 20 \%$ of the initial peak PSD level (i.e., from 30 January). Then within only a few hours between inbound and outbound orbit trajectories on 02 February, the PSD at $\mathrm{L}^{*}>\sim 5.5$ increased drastically by more than an order of magnitude. On 03-04 February, a new peak in PSD had formed at $5.5<\mathrm{L}^{*}<7.5$, though interestingly, at the same time, the PSD from the dropout remnant of 02 February at $\mathrm{L}^{*}<5$ is still clearly visible and nearly unchanged except for some slow decay. The outer belt exhibits a double-peaked structure on 03-04 February, with the peak from the original remnant belt at $L^{*} \sim 4.5$ and the new, enhanced belt at $L^{*} \sim 6.5$. Subsequently, the main phase of the storm occurred between the outbound orbit on 04 February and the inbound on the 05 February, and when the THEMIS spacecraft came back into the outer belt, they observed the results of a massive dropout that had occurred at all $\mathrm{L}^{*}$ down to $\sim 4.5$. During the $\sim 1.25 \mathrm{~h}$ delay between TH-D (first to enter/leave the belt) and TH-E (last to enter/leave it) on the inbound orbit trajectories of 05 February, the peak in PSD between $4<\mathrm{L}^{*}<4.5$ was observed to grow by a factor of $\sim 4$. Around $3 \mathrm{~h}$ later, during the outbound orbit trajectories on 05 February, the PSD had increased by over an order of magnitude out to $\mathrm{L}^{*}>5.5$. The PSD continued to increase on 06 February, exhibiting a fully restored outer belt, dramatically enhanced above the pre-storm levels and peaked at $\mathrm{L}^{*} \sim 5.5$. This new belt exhibited an increase in PSD of over 2 orders of magnitude over a range of $L^{*}$ from approximately 4 to 7 , on a time scale of less than a day. Over the last few days of the period, the broad peaked distribution persisted at approximately the same level, with some finer variations that will be further described in the next section.

[18] Compared to the 04 February storm, equatorially mirroring, relativistic electrons in the outer radiation belt exhibited entirely different behavior during the 11 April 2010 storm. During this period, all three THEMIS spacecraft were in the post- (pre-) midnight MLT sector during their inbound (outbound) orbit trajectories. The initial distribution revealed a very enhanced belt, which was the result of an enhancement during the recovery phase of the 05 April storm. This initial distribution exhibited a peak in PSD at $\mathrm{L}^{*} \sim 6$, though the SST instruments were near their saturation levels at $\mathrm{L}^{*}<\sim 5$ for 07-10 April, so these PSDs (and in particular the local minima in the distribution at around $\mathrm{L}^{*} \sim 4$ ) should not be trusted. This, however, has no effect on the interpretation of the results for this study. Over the first few days of the period, this distribution also underwent some interesting variations, which we do not discuss in detail here. The final pre-storm distribution was that observed by THEMIS on their outbound trajectories on 10 April, which revealed a very broadly peaked or even flat PSD distribution extending to $\mathrm{L}^{*}>8$. The distributions from 11 April were during the SSC and main phase; they revealed inward motion of the distribution with losses starting at higher $\mathrm{L}^{*}$ and moving inward in time. The outbound distributions reveal a very sharp gradient in PSD at $\mathrm{L}^{*} \sim 6$ and a peak in PSD between $4.5<\mathrm{L}^{*}<5.5$. Since the inbound and outbound orbits are all in the tail, this was most likely a temporal change in the distribution and not a result of MLT differences. By the next day, the peak PSD had dropped by another factor of 6 and the peak had moved to $\mathrm{L}^{*}<5$. Interestingly, the PSD increased significantly at $\mathrm{L}^{*}<5$ from 10 to 12 April. Over the remaining days shown in the period, the key features are (1) a slow decay in PSD at $\mathrm{L}^{*}<6,(2)$ an increase in PSD at $L^{*}>6.5$ between 15 and 16 April, and (3) a second peak in PSD at $\mathrm{L}^{*} \sim 6.5$ observed during the outbound trajectory on 14 April. These features, as well as the rest of those discussed here for both storms, will be analyzed in detail in section 4.

\subsection{Distributions of Trapped Versus Precipitating Electrons Observed From LEO}

[19] We can develop a more complete picture of the global distributions of relativistic electrons in the outer belt using POES flux observations from LEO of trapped and precipitating $>1 \mathrm{MeV}$ electrons; these are shown in Figure 4. Note that we have also examined both high time resolution and MLT-binned flux maps to ensure that those shown here are representative of the population and critical details were not lost by bin averaging. From Figure 4, trapped electron fluxes are quite consistent (qualitatively) with the PSD observations derived from the equatorial THEMIS spacecraft, revealing the same variations throughout the course of each storm; however, precipitating electron data reveal important new features. For the February 2011 storm, the outer belt was initially rather quiescent, with a small peak located between L of 4 and 5. A dropout throughout the belt occurred starting on 01 February, and the trapped electrons became enhanced at higher L-shells $(\mathrm{L}>5.5)$ on $03-04$ February. Another, stronger dropout occurred during the main phase of the storm on 05 February, essentially 
a) 2011 Storm: POES >1 MeV Trapped Electrons

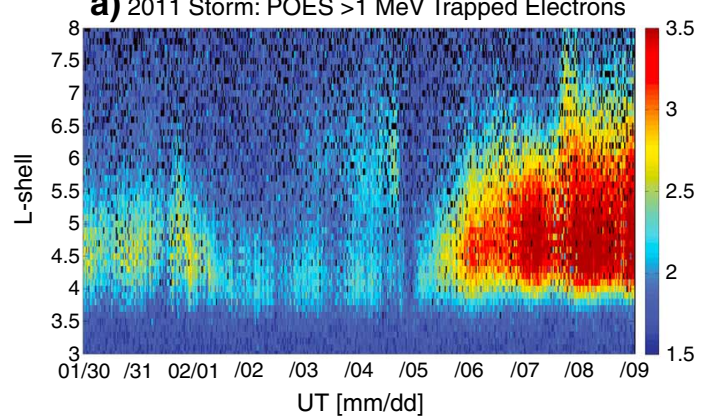

b) 2011 Storm: POES > 1 MeV Precip. Electrons

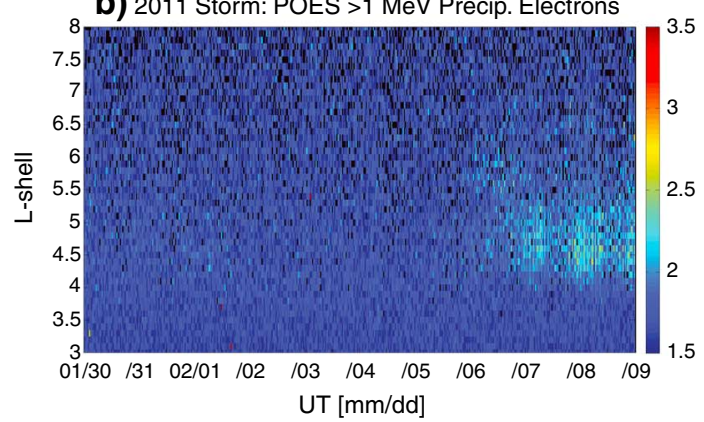

C) 2010 Storm: POES > 1 MeV Trapped Electrons

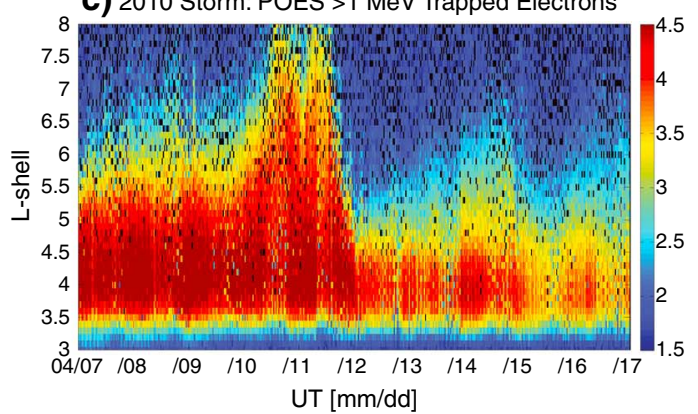

d) 2010 Storm: POES >1 MeV Precip. Electrons

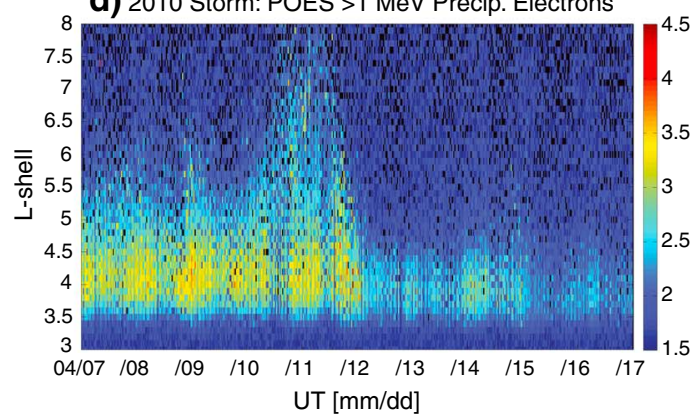

Figure 4. NOAA-POES observations of trapped and precipitating electrons at low-Earth orbit. For the February 2011 storm, trapped (a) and precipitating (b) $>1 \mathrm{MeV}$ electron fluxes $\left(\log _{10}\right.$ of flux, units \#/ $\mathrm{cm}^{2}$-s-sr, shown in color) averaged from six POES spacecraft are shown binned by time $(30 \mathrm{~min})$ and $L^{*}\left(0.1 R_{E}\right)$. (c and d) show the same format for $>1 \mathrm{MeV}$ fluxes during the April 2010 storm.

emptying the belt at POES altitudes (i.e., at low pitch angles). Notably, the precipitating electron distributions (Figure 4b) revealed no enhancements above background levels at any L-shells during either of the dropouts. In the following days, 05-09 February, fluxes of $>1 \mathrm{MeV}$ trapped electrons at LEO were replenished, starting at $4<\mathrm{L}<4.5$ and gradually filling in at higher L-shells in time over the course of a day. During this prolonged recovery phase, the belt became significantly enhanced above pre-storm levels between $4<\mathrm{L}<7.5$. More notably, starting late in the day on 06 February, the precipitating flux observed by POES also began to rise above background levels. The precipitating fluxes were observed between $4<\mathrm{L}<5.5$ and peaked at about $10 \%$ of the simultaneously observed trapped flux population at LEO.

[20] During the development of the storm event resulting in outer belt depletion on 11 April 2010, POES observed an initially enhanced belt spanning $3.5<\mathrm{L}<6$, as seen already on 07 April. This enhanced belt spread out to $\mathrm{L} \sim 8$ or more on 10-11 April before experiencing a main-phase dropout on 11-12 April. This dropout reduced the peak flux levels by $\sim 90 \%$ and the range in trapped fluxes to $3.5<\mathrm{L}$ 5 . The precipitating fluxes throughout the pre-storm period were enhanced above background between $3.5<\mathrm{L}<5.5$ on 07-10 April and extending to higher L-shells on 10-12 April. These precipitating fluxes represented $\sim 10-20 \%$ of their trapped counterparts simultaneously observed at LEO. During the recovery phase, fluxes started to build back up at $\mathrm{L}>5$ until another weak dropout signature around 00:00 UT on 15 April occurred, lowering flux levels throughout the belt. The fluxes remained below their pre-event levels for several days throughout the recovery phase and after the end of the storm. Precipitation fluxes were also above background at $3.5<\mathrm{L}<4.5$ throughout most of the recovery phase. It is also of interest to note that there were flux enhancements of both trapped and precipitating electrons at $\mathrm{L}<7$ at the end of the day on 14 April.

\section{Analysis and Interpretation}

\subsection{Source and Acceleration}

[21] To develop an understanding for the causes of the extreme and very different outer radiation belt variations observed during these two storms, we examined a data set of multi-point wave observations. Concerning acceleration, growing peaks in PSD were observed on 02-04 and 05-07 February 2011 and 14 April 2010. Wave amplitudes of whistler-mode chorus are shown in Figures 5 and 6 for both events as observed by all three THEMIS spacecraft, which all revealed consistent results. For comparison, the observations during the outbound trajectories of each orbit are shown for the February 2011 storm, while for the April 2010 storm, the inbound observations are shown. It is in this format because chorus distributions reveal a strong dependence on MLT [e.g., Li et al., 2009] but for these orbital trajectories during both events, the spacecraft were in the same MLT sector, between 01:00 and 05:00 MLT. Interestingly, on 01-04 February 2011, chorus waves with enhanced amplitudes were observed at $\mathrm{L}^{*}>5.5$, consistent with where the broad peak in PSD formed after the dropout on 01-02 February. Then after the second dropout during the main phase of the storm on 04-05 February, the peak in PSD (Figure 3a) was observed growing first at $3.5<\mathrm{L}^{*}<5$ (inbound) and then at $5<\mathrm{L}^{*}<6$ (outbound) on 05 February and finally as the growing, broadly peaked distribution (peak PSD at $\mathrm{L}^{*} \sim 5.5$ ) on 06-07 February. Based on the chorus observations from THEMIS during the 

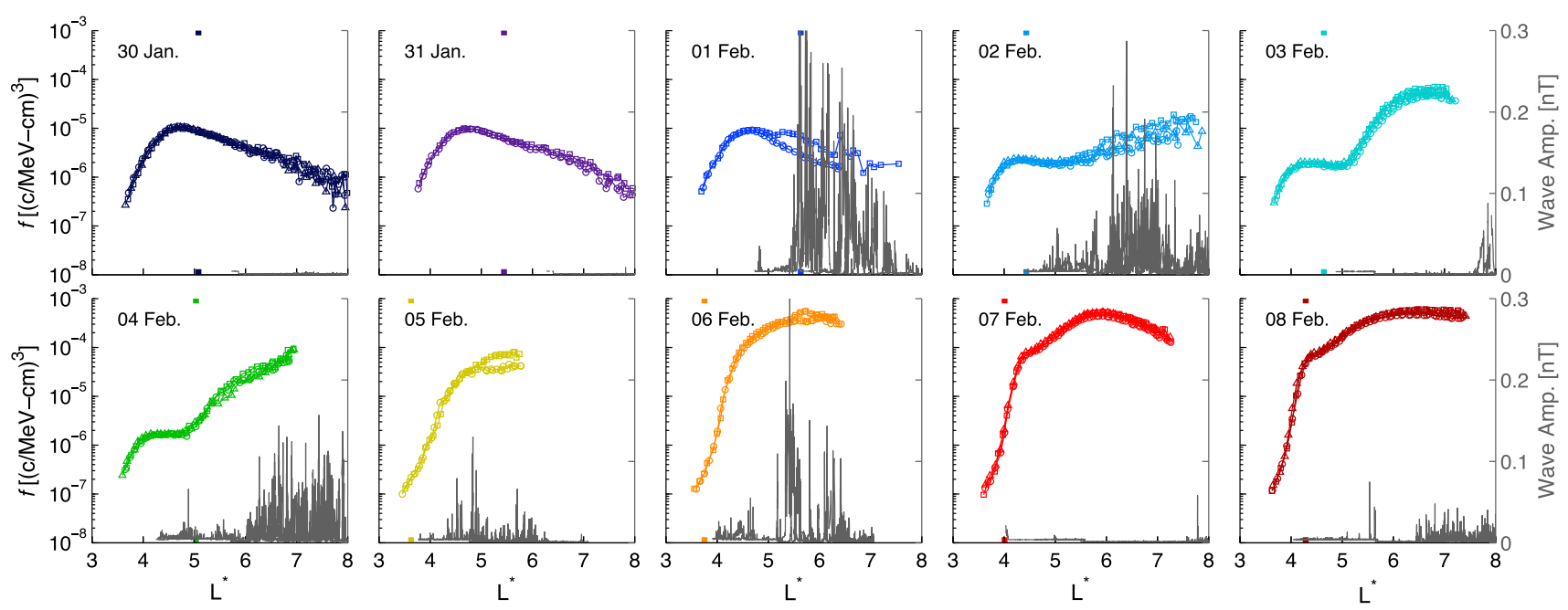

Figure 5. Electron PSD and chorus wave amplitude distributions in $\mathrm{L}^{*}$ observed by TH-A, TH-D, and TH-E during the February 2011 storm. Only observations from the outbound trajectories of the orbit are shown, since these correspond to the 01:00-05:00 MLT sector (i.e., the same as that shown in Figure 6 for the April 2010 event). The different plots show the PSD distribution (corresponding to the left vertical axis) and chorus wave amplitudes (corresponding to the right vertical axis) for each day in the period of interest, as labeled, and for the PSD curves, the colors correspond to those used in Figures 2 and 3. The plasmapause locations, calculated using the O'Brien and Moldwin [2003] model using Dst, are indicated for each day with the corresponding colored bars on the $X$ axis.
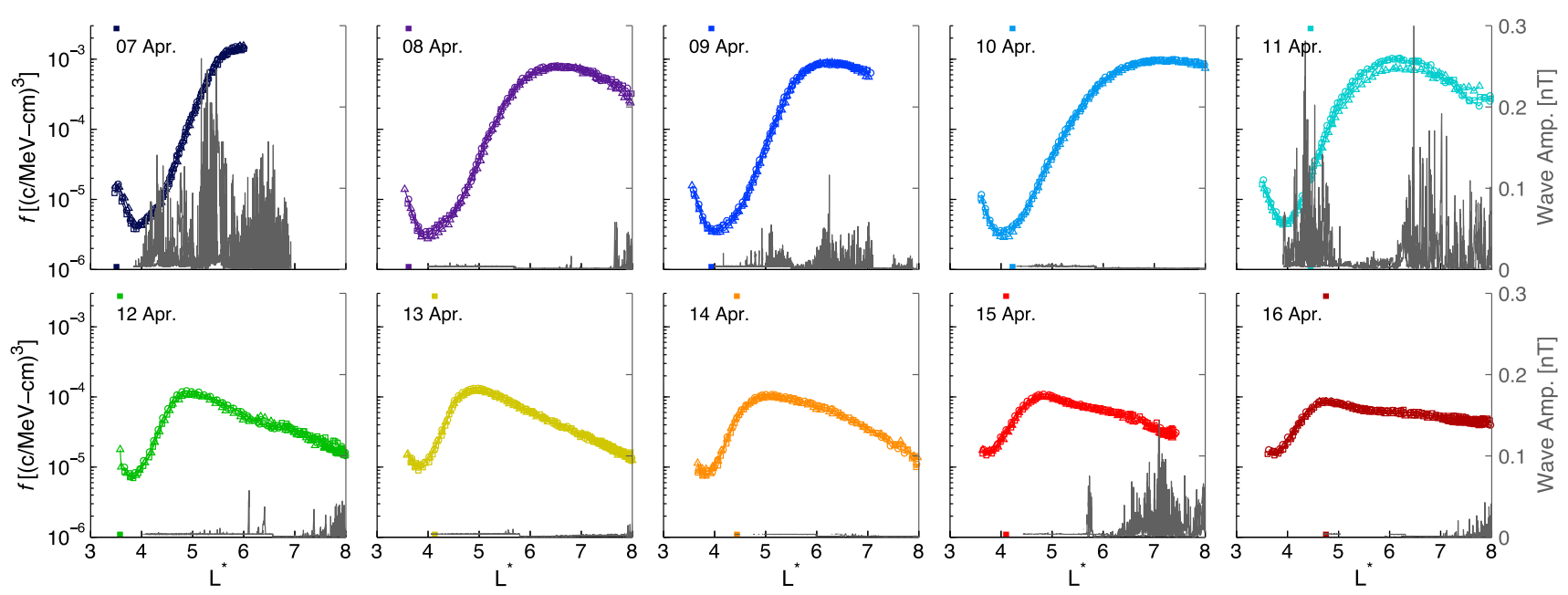

Figure 6. Electron PSD and chorus wave amplitude distributions in L* observed by TH-A, TH-D, and TH-E during the April 2010 storm. Only observations from the inbound trajectories of the orbit are shown, since these correspond to the 01:00-05:00 MLT sector (i.e., the same as that shown in Figure 5 for the February 2011 event). The different plots show the PSD distribution (corresponding to the left vertical axis) and chorus wave amplitudes (corresponding to the right vertical axis) for each day in the period of interest, as labeled, and for the PSD curves, the colors correspond to those used in Figures 2 and 3. The plasmapause locations, calculated using the O'Brien and Moldwin [2003] model using Dst, are indicated for each day with the corresponding colored bars on the $X$ axis.

recovery phase of this storm (i.e., 05-08 February in Figure 5), chorus activity started at $3.5<\mathrm{L}^{*}<5$ on 05 February (most evident in the inbound trajectories, not shown in Figure 5, while THEMIS were in the post-dawn MLT sector; chorus wave amplitudes of $>0.1 \mathrm{nT}$ were observed at $3.5<\mathrm{L}^{*}<5$ during the inbound orbits). The chorus activity subsequently moved out to $5<\mathrm{L}^{*}<7$ on 06 February and then $\mathrm{L}^{*}>5.5$ on 07-08 February (again, the amplitudes were stronger at $\mathrm{L}^{*}>5$ on the post-dawn, inbound trajectories, not shown in Figure 5, for 07-08 February). Electron PSD was enhanced by over two orders of magnitude over a broad range of $\mathrm{L}^{*}$ in less than 1 day from 05 to 06 February. This is consistent with theoretical time scales for acceleration by wave-particle interactions with chorus [e.g., Horne et al., 2005; Summers et al., 


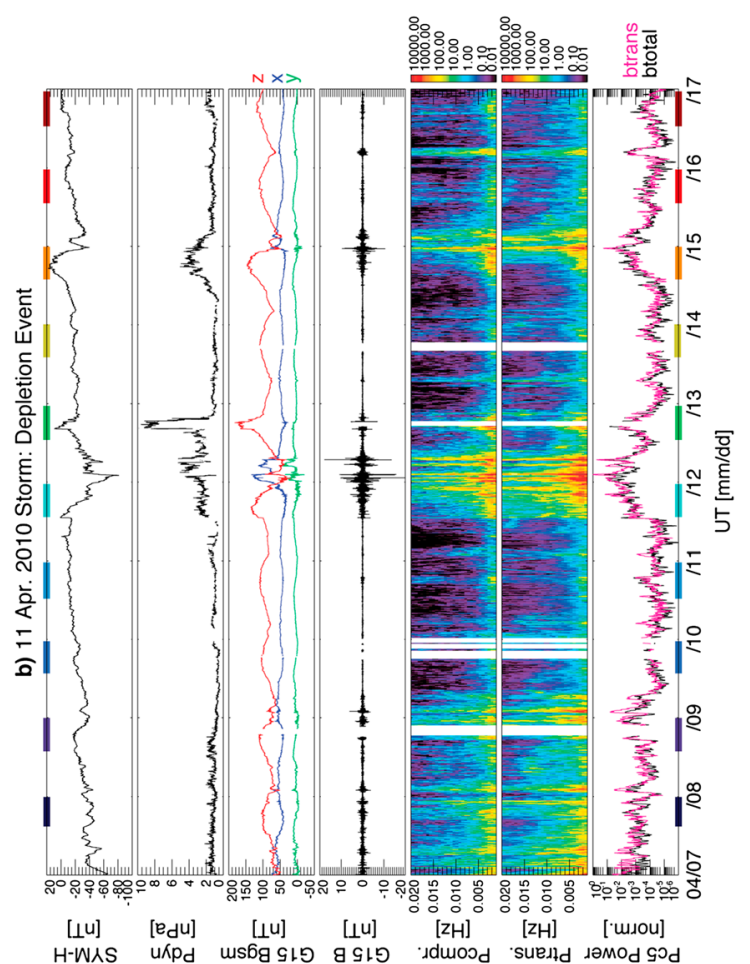

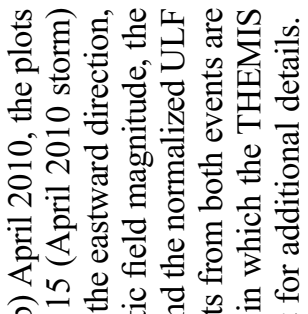

อิ।

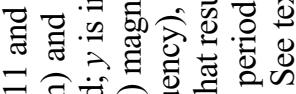

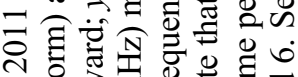

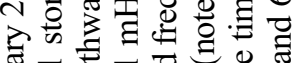

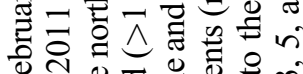

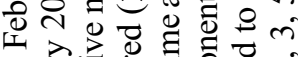

ङ

๔ี

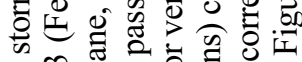

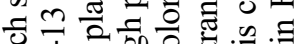

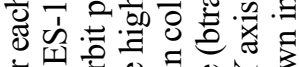

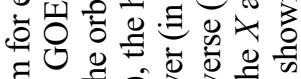

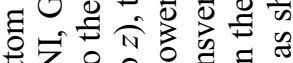

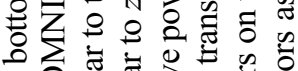

yod

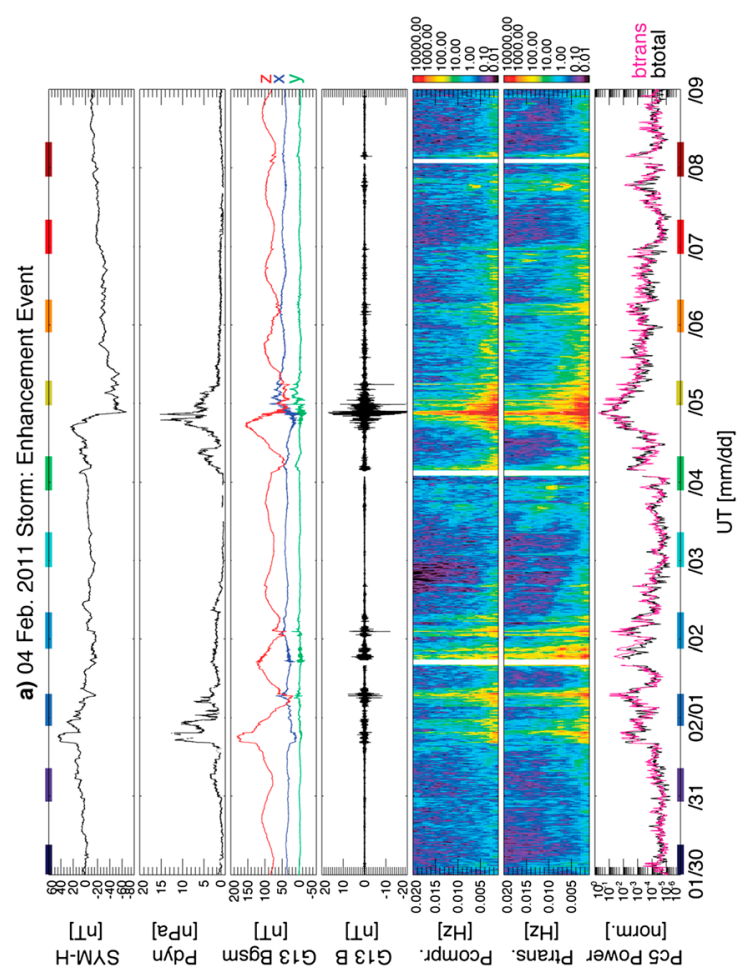

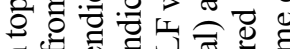

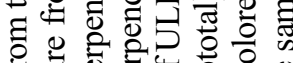

这过触

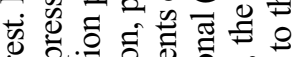

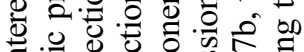

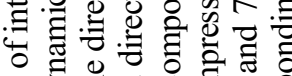

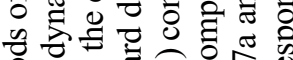

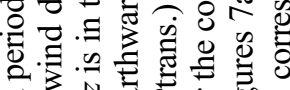

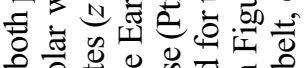

00

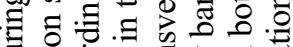

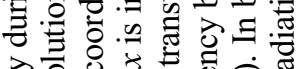

它家

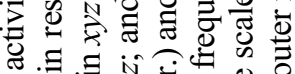

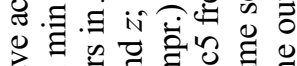

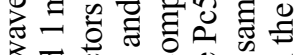

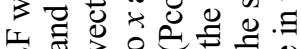

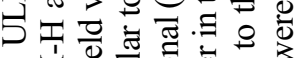

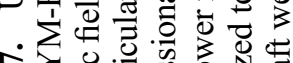

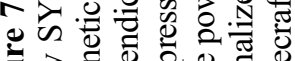

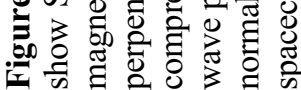


2007], including also when nonlinear interactions are accounted for [Albert, 2002]. Furthermore, here we have examined equatorially mirroring, relativistic electrons that, based on data-derived energy and pitch angle diffusion coefficients and 2- and 3-dimensional diffusion modeling for stochastic wave-particle interactions with chorus [e.g., Horne et al., 2005; Li et al., 2007; Shprits et al., 2009b], are predominantly scattered up in energy and away from the loss cone; such diffusion promotes rapid energization over loss to the atmosphere and generates a local source of electron PSD at corresponding $\mathrm{Mu}$ and $\mathrm{K}$. Summarizing from the February 2011 outer belt enhancement event, growing peaks in phase space density were observed to occur at the same $\mathrm{L}^{*}$ ranges where high-amplitude chorus waves were simultaneously observed. We thus present this as evidence in support of local acceleration of equatorial, relativistic electrons by WPI with whistler-mode chorus.

[22] From Figure 6, the pre-storm period of the April 2010 event exhibited high-amplitude chorus waves intermittently on 07-12 April throughout the entire outer belt. Then during the recovery phase of the storm, chorus activity was only observed at $\mathrm{L}^{*}>6.5$ (with the exception of the small band of activity at $\mathrm{L}^{*} \sim 5.75$ on 15 April), possibly due to lower magnetospheric convection and/or the outward expansion of the plasmasphere. The relative lack of chorus waves in the recovery phase is consistent with the IMF Bz being more positive on average for this event. The peak in PSD observed on 14 April occurred around the same time as the large sub-storm that occurred around 23:20 UT on 14 April, during and after which chorus waves were also observed at $\mathrm{L}^{*}>6.5$. Such a peak may be additional evidence of local chorus acceleration or possibly evidence of an injection of electrons from the plasma sheet producing a relativistic electron response into the region near GEO. Such injections have been observed before [e.g., Ingraham et al., 2001], but the exact details of such an injection process from the plasma sheet remain an outstanding question. Here we speculate on two scenarios. First, there may be some local acceleration process within the plasma sheet itself or at the dipolarization front(s), particularly in the hypothetical scenario in which multiple, localized dipolarization fronts [e.g., Runov et al., 2012; Pembroke et al., 2012] repeatedly energize and recirculate a percentage of electrons [e.g., Gabrielse et al., 2012] until they attain hundreds of $\mathrm{keV}$ in kinetic energy. For this to occur, electrons must gain additional energy at each dipolarization front they encounter; otherwise, the total adiabatic acceleration would simply be reversed. Alternatively, these may represent electrons that originated in the outer radiation belt were transported outward into the plasma sheet by radial diffusion and then were re-injected by the dipolarization front before they could drift across the tail and escape from the system. Further discussion on this injection is beyond the scope of this study, since it appears to have little effect on the subsequent PSD distributions, and it is only briefly remarked upon here as a point of interest.
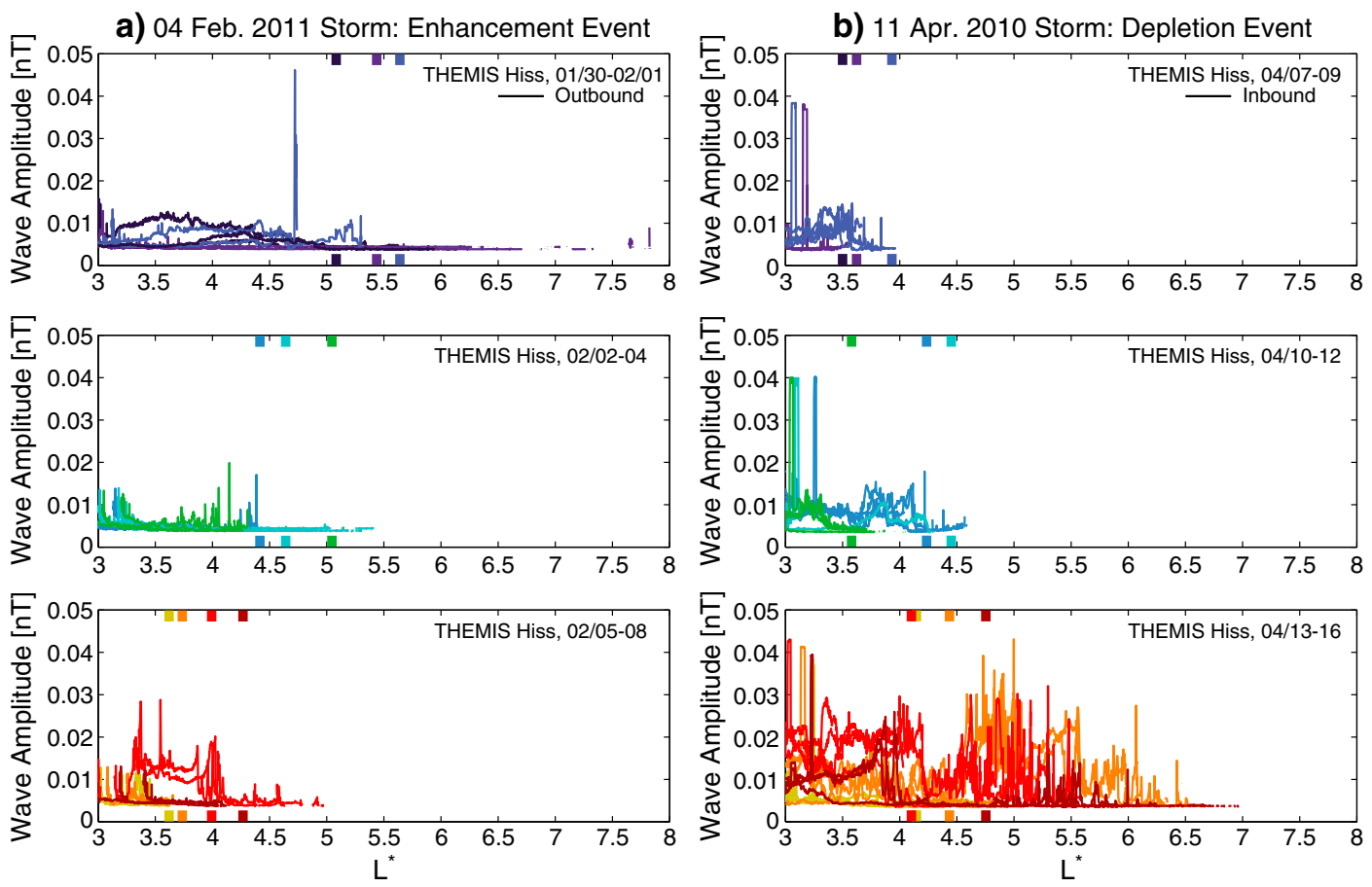

Figure 8. Hiss wave amplitude distributions in L* observed by TH-A, TH-D, and TH-E during (a) the February 2011 storm and (b) the April 2010 storm, with observations from the outbound and inbound trajectories of the orbit shown, respectively, for each storm, since these correspond to the 01:00-05:00 MLT sector during both events. For each event, the top plot shows wave amplitudes from all three spacecraft for days 1-3 of the 10 day period (the corresponding date ranges are labeled on each plot in month/day format), the middle plot for days 4-6, and the bottom plot for days 7-10, each with colors corresponding to those used in Figures 2, 3, and 5-7. The plasmapause locations, calculated using the O'Brien and Moldwin [2003] model using Dst, are indicated for each day with the corresponding colored bars on the $X$ axis. 
[23] Both events exhibited enhancements in ULF wave activity, including in the Pc5 frequency band that can resonate with relativistic electron drift frequencies. GOES observations of these waves are shown in Figure 7 over the periods of interest for both storms. For the February 2011 storm, peak power occurred during the main phase of the storm, on 04-05 February. However, there were other periods of enhanced wave activity in the pre-storm period from 31 January to 02 February, associated with the impact of the first SIR. Associated with the storm itself, the Pc5 ULF wave power remained elevated throughout all of the main phase and most of the recovery period, from 04-07 February. At these times, drift resonant acceleration may have played a role in the PSD enhancements. Peaked PSD distributions in the outer radiation belt are often cited as evidence against acceleration by inward transport from a source in the plasma sheet. However, here we cannot entirely rule out the possibility of peaks developing due to on/off sources of PSD injected from the plasma sheet between observations (i.e., when none of the THEMIS spacecraft were in the outer belt); higher temporal resolution for radial distributions would be necessary to resolve this. Regardless, on-off source from the plasma sheet cannot explain the clearest case of a growing peak in PSD observed by TH-D and TH-E on the inbound trajectories of their orbits on 05 February. Also, the outbound distributions that reveal maximum PSD at highest $\mathrm{L}^{*}$ (e.g., 02/02, 02/03, 02/04, and $02 / 05$ in Figure $3 b$ ) are either a result of $L^{*}$ not being defined by the Tsyganenko and Sitnov [2005] model or the required energy range to conserve $\mathrm{Mu}$ extending below that of the coincidence logic energy channels of the SST electron instruments [see Turner et al., 2012b]; when all of the electron channels are used to calculate PSD at these higher L-shells, extending the energy range to $\sim 30 \mathrm{keV}$, the observed PSD distributions beyond this boundary (not shown) are actually peaked. For the April 2010 storm, the background wave power was weaker than that during the enhancement event, and other than at the beginning of the period (i.e., during the recovery phase of the 05 April storm), the ULF power occurred over shorter periods. Like the
2011 event, the enhancements in ULF wave activity occurred during periods of enhanced solar wind dynamic pressure and/or fast solar wind speed. PSD distributions do not suggest that inward radial diffusion was the major acceleration mechanism during this event, except perhaps between $14-16$ April at $\mathrm{L}^{*}<4.5$.

\subsection{Loss}

[24] Concerning losses, whistler-mode hiss and EMIC waves were also observed during both storm periods. Figure 8 shows hiss wave amplitudes observed by the three THEMIS spacecraft. Note that the locations agree approximately with the plasmapause locations shown, with hiss being observed primarily within the plasmasphere. During the first 6 days of the February 2011 storm, hiss waves were observed out to $L^{*} \sim 5.5$, which may explain the continual slow decay observed there between TH-D and TH-E (since TH-D passed through the belt around $1 \mathrm{~h}$ before TH-E did) and from day to day during the periods 30-31 January and 02-04 February. Stronger hiss waves were also evident in the outbound trajectories in the same $\mathrm{L}^{*}$ range on these days, when THEMIS spacecraft were in the pre-midnight MLT sector (not shown in Figure 8a). During the main phase and beginning of the recovery phase of the storm, hiss was restricted to $L^{*}<3.5$, consistent with the strong erosion of the plasmasphere. During the rest of the recovery phase, hiss was observed with higher amplitudes mostly at $\mathrm{L}^{*}<4.5$, which corresponded to the inner edge of the outer belt observed there. During the April 2010 storm, hiss was restricted to $L^{*}<4.5$ during the first 7 days of the period shown. Then during the late recovery phase and remainder of the period from 14 to 16 April, high-amplitude hiss waves were observed out to $L^{*} \sim 6.5$, which is consistent with the slow decay of PSD observed at $\mathrm{L}^{*}<6.5$ during these last few days.

[25] Figure 9 shows L-shells and times at which EMIC waves were observed by the THEMIS ground magnetometers and GOES during the periods of interest. Comparing the two storms, it is immediately clear that there were more EMIC waves during the April 2010 depletion event.
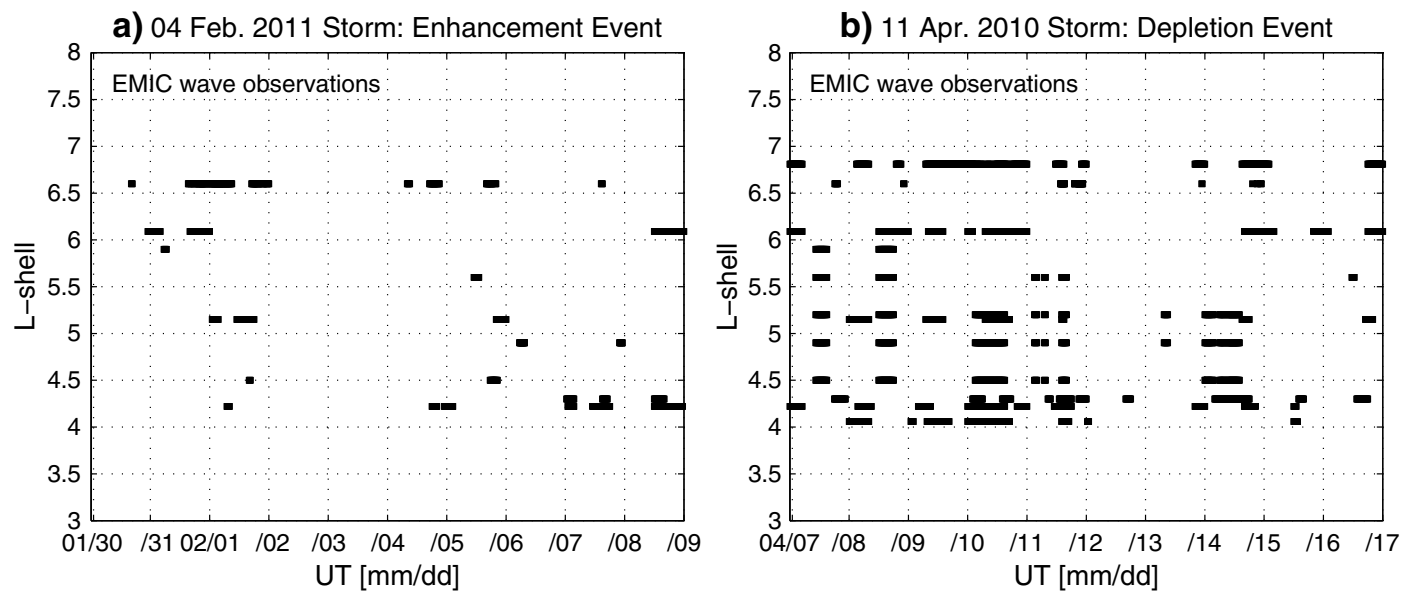

Figure 9. Locations in L and durations of EMIC waves observed by CARISMA, Sodankyla, and Halley Bay ground magnetometers and GOES spacecraft at GEO for (a) the February 2011 storm and (b) the April 2010 storm. 
During the pre-storm period of that event (07-11 April), waves in the EMIC frequency band were observed regularly over a broad range of L-shells throughout the outer belt. Interestingly, the wave activity was not observed during the main phase and start of the recovery phase starting late on 11 April and lasting throughout 12 April, when the dropout in PSD occurred. It should be noted, however, that most of these EMIC observations are ground based, and it has been reported that EMIC waves are more likely to be observed on the ground several days into storm recovery compared to the main and early recovery phases [e.g., Bortnik et al., 2008]. Activity picked up again over a broad range of L-shells around the end of the day on 13 April. The February 2011 storm on the other hand revealed fewer EMIC waves, and those that were observed occurred over shorter periods and were more localized in L. EMIC waves may have played a role in loss during the recovery phase of this event, however. The bite out of the PSD distribution at $4.3<\mathrm{L}^{*}<5$ on $07-08$ February seems most consistent with loss by EMIC waves, since (i) the plasmapause was located at $\mathrm{L}<4.3$, (ii) observed hiss waves were limited to $\mathrm{L}<4$, (iii) EMIC waves were observed by ground magnetometers at $4<\mathrm{L}<5$ (most consistently around $\mathrm{L} \sim 4.25$ ) from 07 to 09 February, and (iv) there was enhanced precipitation of $>1 \mathrm{MeV}$ electrons into the atmosphere at $4<\mathrm{L}$ 5 on 07-09 February. However, we cannot rule out the possibility that the precipitation may have alternatively just been the result of the increase in the total trapped population.

[26] Each of the dropouts observed during these periods was associated with sudden enhancements of solar wind dynamic pressure, i.e., on 31 January-02 February and 04-05 February 2011 and 11-12 April 2010. The behavior of the PSD distributions during these dropouts, particularly the 31 January-02 February and 11-12 April periods, was consistent with what is expected from a combination of magnetopause shadowing at high $\mathrm{L}^{*}$ followed by rapid outward radial transport. Both cases exhibited losses first at higher $\mathrm{L}^{*}$ and then later at lower $\mathrm{L}^{*}$, evidenced by the fact that the peak in PSD was falling in magnitude with inward motion in $\mathrm{L}^{*}$ [see Turner et al., 2012a]. The 11-12 April period was particularly revealing, showing the initial distribution on the inbound trajectory on 11 April followed by the distribution on the outbound (only a couple hours later), which exhibited (i) a very sudden drop in PSD at $\mathrm{L}^{*} \sim 6$ (consistent with the discontinuous signature of magnetopause shadowing), (ii) a peak that decreased and moved in from $L^{*} \sim 6$ to $L^{*} \sim 5$ (possibly due to a combination of the initial shock-like injection [e.g., Li et al., 1998] and subsequent inward transport), and (iii) an increase in PSD at $\mathrm{L}^{*}$ inside of the peak location between 11 and 12 April (consistent with inward diffusion away from the remnant peak in PSD after magnetopause shadowing occurred). ULF activity was enhanced during all three dropout periods, which is a requirement for breaking the third invariant and enabling rapid transport across drift shells. Since EMIC waves are often generated during magnetospheric compressions [e.g., Usanova et al., 2008; 2012, and references therein], we also considered the possibility of EMIC-related loss of MeV electrons to the atmosphere. Numerical simulations have also shown that electron scattering by EMIC waves with sufficient wave amplitudes should result in strong diffusion [e.g., Shprits et al., 2009a]. Also, previous model-data comparisons have shown that loss timescales of relativistic electron precipitation into the atmosphere during the main phase of storms can be on the order of several hours [e.g., Selesnick, 2006; Tu et al., 2010] to around a day [e.g., Thorne et al., 2005]. However, the precipitating flux maps reveal no significant increase in losses to the atmosphere that would support the depletion of the trapped population. Even in the absence of strong diffusion, some enhancement in precipitating flux throughout the loss cone would be expected if the majority of the electrons in the belt were being stochastically scattered into the atmosphere, and to properly estimate loss rates to the atmosphere, model-data comparisons should be conducted along the same lines as previous studies [e.g., Thorne et al., 2005; Selesnick, 2006; Tu et al., 2010]. Based on the lack of enhanced precipitating electrons and the observed evolution of the PSD distributions shown here, we conclude that these dropouts were more consistent with the model of magnetopause shadowing and subsequent enhanced outward radial transport, as discussed in Turner et al. [2012a, and references therein].

\subsection{Transport}

[27] As discussed above, transport across drift shells appeared to play an important role in dropout-related losses that occurred during each of these storm periods. Diffusive radial transport was also evident at various times as well. For example, note the inward spreading of PSD observed at $\mathrm{L}^{*}<4.5$ during both active recovery periods, from 06 to 08 February 2011 and 14 to 16 April 2010 (Figures 3a and $3 b)$. Outward transport away from the peaks in PSD [e.g., Reeves et al., 1998; Miyoshi et al., 2003] was also observed during these two recovery phase intervals. The PSD increased at $L^{*}>6$ from 07 to 08 February 2011 and also at $\mathrm{L}^{*}>5$ from 15 to 16 April 2010 consistent with outward radial transport. Particularly evident in the April 2010 case, the PSD distribution exhibited flattening at higher $L^{*}$. This flattening is consistent with our expectation from theory given the expected stronger radial diffusion at higher $\mathrm{L}^{*}$, an initially peaked distribution, and assuming sufficient source population to balance the loss from outward transport. We also examined ULF wave data from THEMIS (not shown), which was consistent with that observed by GOES but revealed that ULF wave activity extended to L-shells beyond GEO, further supporting strong radial diffusion there. From the chorus observations (Figures 5 and 6) revealing strong chorus wave amplitudes at $\mathrm{L}^{*}>6$ in both of these periods, it should be noted that these enhancements in PSD were probably the result of a combination of chorus acceleration and outward diffusion (enhanced ULF wave activity during these periods allowed for enhanced outward diffusion). This presents an interesting scenario in which outward transport may serve as a regulating mechanism of the system; i.e., WPI may be producing peaks in PSD during active periods, but these newly accelerated relativistic electrons will also experience enhanced diffusion outwards in the active conditions and thus be decelerated and potentially lost from the system.

\section{Conclusions}

[28] We have identified a database of geomagnetic storms and evaluated their effects on the PSD of equatorially mirroring, relativistic electrons throughout the outer radiation belt. From this database, we have analyzed two storms in detail, one that resulted in a net enhancement of PSD in 
the outer belt and another that resulted in a depletion of PSD throughout the outer belt. For each event, we examined multi-point observations of solar wind and geomagnetic conditions; $\mathrm{L}^{*}$-distributions of electron PSD for $\mathrm{Mu}=1000$ $\mathrm{MeV} / \mathrm{G}$ and $\mathrm{K} \sim 0 \mathrm{G}^{1 / 2} \mathrm{R}_{\mathrm{E}}$ (i.e., relativistic and equatorially mirroring); trapped and precipitating $>1 \mathrm{MeV}$ fluxes from LEO; and chorus, hiss, EMIC, and ULF waves observed throughout the system. From these observations, we have presented PSD distribution variations that are consistent with (i) local acceleration by WPI between relativistic electrons and whistler-mode chorus based on growing peaks in PSD directly corresponding to peak locations in chorus wave amplitudes; (ii) dropout events resulting from magnetopause shadowing combined with enhanced outward radial transport; (iii) slow, decay-like loss associated with hiss; (iv) precipitation loss corresponding to the location of observed EMIC waves and a reduction in equatorial PSD; and (v) radial diffusion away from peaks in PSD during active recovery phase conditions. It should be noted that these are all observations from only two storms, and more rigorous, statistical studies as well as additional case studies using multi-point observations (incorporating THEMIS, POES, GOES, and soon benefiting from the addition of the recently launched, twin Van Allen Probes, or RBSP, spacecraft) will confirm whether these cases represent general behavior in the outer belt. With this in mind, the main conclusions of this study are as follows:

[29] 1. When compared to the storm that resulted in a depletion of PSD throughout the belt, the storm that resulted in an overall PSD enhancement exhibited (i) more enhanced chorus wave amplitudes, (ii) broader ranges in $\mathrm{L}^{*}$ of chorus activity, (iii) a smaller range in $\mathrm{L}^{*}$ of hiss during the recovery phase, (iv) fewer EMIC wave events throughout the period, and (v) more prolonged periods of enhanced Pc5 ULF wave activity. Both storms also provided evidence of radial diffusion, both inward and outward from a peak in PSD within the outer belt itself, as well as losses to the atmosphere corresponding to observed locations of EMIC waves and slower decay rates during quiet times, possibly corresponding to plasmaspheric hiss.

[30] 2. We observed evidence consistent with rapid acceleration by WPI between whistler-mode chorus and relativistic electrons; PSD peaks grew in time on the same $\mathrm{L}^{*}$ as enhancements in chorus wave amplitude were observed. The PSD peaks and chorus waves were consistently beyond the plasmapause location, even as it evolved over the course of the storms. However, we cannot fully rule out the role of on/off source due to sudden injections of electrons from the plasma sheet [e.g., Ingraham et al., 2001; Lui et al., 2012], since distributions at higher time cadence are necessary to resolve them. Regardless, we showed that the source mechanism can increase the PSD throughout the entire belt by more than two orders of magnitude in less than 1 day.

[31] 3. Dropouts throughout most/all of the outer belt followed after solar wind dynamic pressure enhancements and exhibited features consistent with magnetopause shadowing and subsequent enhanced outward radial transport.

[32] 4. We showed evidence supporting the fact that the outer belt can rebuild seemingly independently of the remnant distribution from a dropout event resulting in a double-peaked distribution in the outer belt, as occurred on 01-04 February
2011. This was not an isolated occurrence; of the 53 storms examined, 13 cases revealed this remnant, double-peaked feature in the post-dropout PSD distributions. Also, such a feature has now been simultaneously confirmed with an independent study using observations from NASA's Van Allen Probes mission (Baker, D. N. et al., A long lived relativistic electron storage ring embedded within the Earth's outer Van Allen radiation zone, Science, in press).

[33] 5. Of the 53 storms in our database, $58 \%$ resulted in an electron PSD enhancement, $17 \%$ resulted in PSD depletion, and $25 \%$ resulted in no significant change. These results are consistent with those of Reeves et al. [2003] and suggest that the set of storms studied here are a representative sample of the ones studied during the previous solar cycle. This also reveals that the variation in the fluxes at GEO follow the variation of the peak in PSD, and, L- or pitch angle dependent variations, particularly increases at lower L-shells during dropouts, may explain the discrepancy with the more recent statistics of Zhao et al. [2013].

[34] This study is a comprehensive assembly of particle and wave data in Earth's outer radiation belt, which affords us the first clear pictures of the various processes responsible for the extreme variations of the relativistic electron populations observed during two similar Dst storms, which display very different radiation belt responses, side by side. Our interpretations of these observations suggest that the current theories involving source, loss, and transport mechanisms in the outer radiation belt are sufficient to explain most of the significant changes in PSD during these two events. The resultant outer belt enhancement or depletion of any particular storm or event is thus the combined effect of these individual processes, acting in concert to produce the observed effects. However, several outstanding questions still remain. The effect of chorus on the PSD distributions shown here is not linearly related to the amplitude of the observed chorus waves, and we must develop a clearer picture of the exact nature of these WPI. This is also true of the slow decay due to hiss and is probably also the case for WPI with EMIC waves, indicating that a better understanding of the radiation belt response to waves of given amplitude is needed. The role of injections of relativistic electrons during sub-storms is still not understood; do the electrons originate in the plasma sheet due to some acceleration mechanism acting there, or do they originate in the outer belt and are just being reintroduced after outward transport into the tail? We also do not currently have a good understanding of the role of Shabansky drift orbits, both in generating chorus and EMIC waves [e.g., McCollough et al., 2012] and in scattering electrons in $\mathrm{K}$ and $\mathrm{L}^{*}$ [e.g., Ukhorskiy et al., 2011]. Here we have also not presented any evidence of magnetosonic wave activity (since the spacecraft were not in the preferred dayside MLT sector and THEMIS FFF data, necessary for identifying magnetosonic waves, were unavailable for the April 2010 event) or the cross L-shell effects of drift-resonant interactions with ULF waves (since PSD as a function of energy must be examined). This study demonstrates the importance and potential of employing multi-point observations from spacecraft (and ground stations and/or balloons) throughout the outer belt system to develop a more comprehensive picture of the entire system. To address these outstanding questions and to continue development towards a full understanding of Earth's outer radiation belt electrons in the near 
future, observational studies should take full advantage of the unprecedented array of particle and wave observatories currently in place for studying the outer belt system, including those based on Earth (e.g., CARISMA, THEMIS-GMAGs, and BARREL), those at LEO (e.g., POES, SAMPEX, and CubeSats like CSSWE and FIREBIRD), and those near the magnetic equator (e.g., Van Allen Probes in the heart of the belt, GOES at GEO, and THEMIS throughout the belt and beyond it into the plasma sheet).

[35] Acknowledgments. We would like to thank the THEMIS mission and instrument teams and the development teams for the IRBEM library (http:// craterre.onecert.fr/prbem/irbem/description.html) and SpacePy tools (http:// spacepy.lanl.gov/). We acknowledge and kindly thank H. J. Singer for GOES data and the Sodankylä Geophysical Observatory for making quick look ground magnetometer data available. The Halley Bay station is funded by the U.S. National Science Foundation grant ANT-1142045 to Augsburg College. CARISMA is operated by the University of Alberta, funded by the Canadian Space Agency, and special thanks to D. K. Milling and A. Kale for the deployment of the new CARISMA induction coil array. I. R. Mann, M. Usanova, and D. L. Turner are thankful for support from the Monitoring, Analyzing, and Assessing the Radiation Belt Loss and Energization (MAARBLE) project funded under the European Commision's (EC) FP7 framework (Note that this work reflects the authors' views, and the EC is not liable for any use that may be made of the information contained herein). I. R. Mann is also supported by a Discover Grant from Canadian NSERC, and M. Usanova is partially supported by the Canadian Space Agency. J. Bortnik and W. Li are thankful for support from a NASA grant (NNX11AD75G). Finally, D. L. Turner is also very thankful for funding to conduct this research from NASA's THEMIS mission (contract NAS5-02099) and a NASA grant (NNX12AJ55G).

\section{References}

Abel, R. W., and R. M. Thorne (1998a), Electron scattering loss in Earth's inner magnetosphere: 1. Dominant physical processes, J. Geophys. Res., 103(A2), 2385-2396.

Abel, R. W., and R. M. Thorne (1998b), Electron scattering loss in Earth' inner magnetosphere: 2. Sensitivity to model parameters, J. Geophys. Res., 103(A2), 2397-2408.

Albert, J. M. (2002), Nonlinear interaction of outer zone electrons with VLF waves, Geophys. Res. Lett., 29, 1275, doi:10.1029/2001GL013941.

Albert, J. M. (2003), Evaluation of quasi-linear diffusion coefficients for EMIC waves in a multispecies plasma, J. Geophys. Res., 108, 1249, doi:10.1029/2002JA009792.

Angelopoulos, V. (2008), The THEMIS Mission, Space Sci. Rev., 141, 5-34.

Auster, U., et al. (2008), The THEMIS fluxgate magnetometer, Space Sci. Rev., 141, 235-264.

Baker, D. N., et al. (1998), A strong CME-related magnetic cloud interaction with the Earth's magnetosphere: ISTP observations of rapid relativistic electron acceleration on May 15, 1997, Geophys. Res. Lett., 25, 2975-2978.

Baker, D. N. (2002), How to cope with space weather, Science, 297, 1486,2002

Bortnik, J., R. M. Thorne, T. P. O’Brien, J. C. Green, R. J. Strangeway, Y. Y. Shprits, and D. N. Baker (2006), Observation of two distinct, rapid loss mechanisms during the 20 November 2003 radiation belt dropout event, J. Geophys. Res., 111, A12216, doi:10.1029/2006JA011802.

Bortnik, J., and R. M. Thorne (2007), The dual role of ELF/VLF chorus waves in the acceleration and precipitation of radiation belt electrons, J. Atmos. Solar Terr. Phys., 69, 378-386.

Bortnik, J., J. W. Cutler, C. Dunson, T. E. Bleier, and R. L. McPherron (2008), Characteristics of low-latitude Pc1 pulsations during geomagnetic storms, J. Geophys. Res., 113, A04201, doi:10.1029/2007JA012867.

Chen, Y., et al. (2007), The energization of relativistic electrons in the outer Van Allen radiation belt, Nat. Phys., 3, 614-617.

Elkington, S. R., M. K. Hudson, and A. A. Chan (2003), Resonant acceleration and diffusion of outer zone electrons in an asymmetric geomagnetic field, J. Geophys. Res., 108, 1116, doi:10.1029/2001JA009202.

D. S. Evans and M. S. Greer (2004), Polar Operational Environment Satellite Space Environment Monitor-2: Instrument descriptions and archive data documentation, NOAA Tech. Mem., 93, 47, version 1.4.

Friedel, R. H. W., G. D. Reeves, and T. Obara (2002), Relativistic electron dynamics in the inner magnetosphere: A review, J. Atmos. Solar Terr. Phys., 64 (2), 265.
Fujimoto, M., and Nishida, A.,(1990), Energization and anisotropization of energetic electrons in the Earth's radiation belt by the recirculation process, J. Geophys. Res., 95, 4265-4270.

Gabrielse, C., V. Angelopoulos, A. Runov, and D. L. Turner (2012), The effects of transient, localized electric fields on equatorial electron acceleration and transport towards the inner magnetosphere, J. Geophys. Res., doi:10.1029/2012JA017873.

Green, J. C., and M. G. Kivelson (2004), Relativistic electrons in the outer radiation belt: Differentiating between acceleration mechanisms, J. Geophys. Res., 109, A03213.

Gonzalez, W. D., B. T. Tsurutani, and A. L. Clua de Gonzalez (1999), Interplanetary origin of geomagnetic storms, Space Sci. Rev., 88, 529-562.

Horne, R. B., and R. M. Thorne (1998), Potential waves for relativistic electron scattering and stochastic acceleration during magnetic storms, Geophys. Res. Lett., 25(15), 3011-3014, doi:10.1029/98GL01002.

Horne, R. B., and R. M. Thorne (2003), Relativistic electron acceleration and precipitation during resonant interactions with whistler-mode chorus, Geophys. Res. Lett., 30(10), 1527, doi:10.1029/2003GL016973.

Horne, R. B., R. M. Thorne, S. A. Glauert, J. M. Albert, N. P. Meredith, and R. R. Anderson (2005), Timescale for radiation belt electron acceleration by whistler mode chorus waves, J. Geophys. Res., 110, A03225, doi:10.1029/2004JA010811.

Horne, R. B., et al. (2007), Electron acceleration in the Van Allen radiation belts by fast magnetosonic waves, Geophys. Res. Lett., 34, L17107.

Hudson, M. K., S. R. Elkington, J. G. Lyon, and C. C. Goodrich (2000), Increase in relativistic electron flux in the inner magnetosphere: ULF wave mode structure, Adv. Space Res., 25 (12), 2327.

Ingraham, J. C., T. E. Cayton, R. D. Belian, R. A. Christensen, R. H. W. Friedel, M. M. Meier, G. D. Reeves, and M. Tuszewski (2001), Substorm injection of relativistic electrons to geosynchronous orbit during the great magnetic storm of March 24, 1991, J. Geophys. Res., 106(A11), 25,759-25,776, doi:10.1029/2000JA000458.

Kamide, Y., W. Baumjohann, I. A. Daglis, W. D. Gonzalez, M. Grande, J. A. Joselyn, R. L. McPherron, J. L. Phillips, G. D. Reeves, G. Rostoker, A. S. Sharma, H. J. Singer, B. T. Tsurutani, and V. M. Vasyliunas (1998), Current understanding of magnetic storms: Storm-substorm relationships, J. Geophys. Res., 103, 17705-17728.

Kellerman, A. C. and Y. Y. Shprits (2012), On the influence of solar wind conditions on the outer-electron radiation belt, J. Geophys. Res., 117, A05217, doi:10.1029/2011JA017253.

Kim, H.-J., and A. A. Chan (1997), Fully adiabatic changes in storm time relativistic electron fluxes, J. Geophys. Res., 102(A10), 22,107-22,116, doi:10.1029/97JA01814.

Lam, M. M., R. B. Horne, N. P. Meredith, S. A. Glauert, T. Moffat-Griffin, and J. C. Green (2010), Origin of energetic electron precipitation > 30 $\mathrm{keV}$ into the atmosphere, J. Geophys. Res., 115, A00F08, doi:10.1029/ 2009JA014619.

Li, W., Y. Y. Shprits, and R. M. Thorne (2007), Dynamic evolution of energetic outer zone electrons due to wave-particle interactions during storms, J. Geophys. Res., 112, A10220, doi:10.1029/2007JA012368.

Li, W., R. M. Thorne, V. Angelopoulos, J. Bortnik, C. M. Cully, B. Ni, O. LeContel, A. Roux, U. Auster, and W. Magnes (2009), Global distribution of whistler-mode chorus waves observed on the THEMIS spacecraft, Geophys. Res. Lett., 36, L09104, doi:10.1029/ 2009 GL037595.

Li, W., R. M. Thorne, J. Bortnik, Y. Nishimura, V. Angelopoulos, L. Chen, J. P. McFadden, and J. W. Bonnell (2010), Global distributions of suprathermal electrons observed on THEMIS and potential mechanisms for access into the plasmasphere, J. Geophys. Res., 115, A00J10, doi:10.1029/2010JA015687

Li, X., D. N. Baker, M. Temerin, D. Larson, R. P. Lin, G. D. Reeves, M. Looper, S. G. Kanekal, and R. A. Mewaldt (1997), Are energetic electrons in the solar wind the source of the outer radiation belt?, Geophys. Res. Lett., 24(8), 923-926, doi:10.1029/97GL00543.

Li, X., D. N. Baker, M. Temerin, T. Cayton, G. D. Reeves, T. Araki, H. Singer, D. Larson, R. P. Lin, and S. G. Kanekal (1998), Energetic electron injections into the inner magnetosphere during the Jan. 10-11, 1997 magnetic storm, Geophys. Res. Lett., 25(14), 2561-2564, doi:10.1029/ 98GL00036.

Loto'aniu, T. M., H. J. Singer, C. L. Waters, V. Angelopoulos, I. R. Mann, S. R. Elkington, and J. W. Bonnell (2010), Relativistic electron loss due to ultralow frequency waves and enhanced outward radial diffusion, $J$. Geophys. Res., 115, A12245, doi:10.1029/2010JA015755.

Lui, A. T. Y., Q.-G. Zong, C. Wang, and M. W. Dunlop (2012), Electron source associated with dipolarization at the outer boundary of the radiation belts: Non-storm cases, J. Geophys. Res., 117, A10224, doi:10.1029/2012JA018084.

Lyons, L. R., R. M. Thorne, and C. F. Kennel (1972), Pitch-angle diffusion of radiation belt electrons within the plasmasphere, J. Geophys. Res., 77 (19), 3455-3474, doi:10.1029/JA077i019p03455. 
Mann, I. R., T. P. O’Brien, and D. K. Milling (2004), Correlations between ULF wave power, solar wind speed, and relativistic electron flux in the magnetosphere: Solar cycle dependence, J. Atmos. Solar Terr. Phys., 66, 187, doi:10.1016/j.jastp.2003.10.002.

Mann, I. R., et al., (2008), The updated CARISMA magnetometer array in the THEMIS Era, Space Sci Rev., 141, pp. 413-451.

McCollough, J. P., S. R. Elkington, and D. N. Baker (2012), The role of Shabansky orbits in compression-related electromagnetic ion cyclotron wave growth, J. Geophys. Res., 117, A01208, doi:10.1029/2011JA016948.

Meredith, N. P., M. Cain, R. B. Horne, R. M. Thorne, R. R. Anderson, and R. R. Anderson (2003), Evidence for chorus-driven electron acceleration to relativistic energies from a survey of geomagnetically disturbed periods, J. Geophys. Res., 108, 1248, doi:10.1029/2002JA009764.

Meredith, N. P., R. B. Horne, R. H. A. Iles, R. M. Thorne, D. Heynderickx, and R. R. Anderson (2002), Outer zone relativistic electron acceleration associated with substorm-enhanced whistler mode chorus, J. Geophys. Res., 107, 1144, doi:10.1029/2001JA900146.

Meredith, N. P., R. B. Horne, S. A. Glauert, D. N. Baker, S. G. Kanekal, and J. M. Albert (2009), Relativistic electron loss timescales in the slot region, J. Geophys. Res., 114, A03222, doi:10.1029/2008JA013889.

Meredith, N. P., R. B. Horne, M. M. Lam, M. H. Denton, J. E. Borovsky, and J. C. Green (2011), Energetic electron precipitation during high-speed solar wind stream driven storms, J. Geophys. Res., 116, A05223, doi:10.1029/2010JA016293.

Millan, R. M., R. P. Lin, D. M. Smith, K. R. Lorentzen, and M. P. McCarthy (2002), X-ray observations of $\mathrm{MeV}$ electron precipitation with a balloon-borne germanium spectrometer, Geophys. Res. Lett., 29(24), 2194, doi:10.1029/2002GL015922.

Millan, R. and R. M. Thorne (2007), Review of radiation belt relativistic electron losses, J. Atmos. Solar Terr. Phys. 69, 362-377.

Millan, R. M., K. B. Yando, J. C. Green, and A. Y. Ukhorskiy (2010), Spatial distribution of relativistic electron precipitation during a radiation belt depletion event, Geophys. Res. Lett., 37, L20103, doi:10.1029/ 2010GL044919.

Miyoshi, Y., A. Morioka, T. Obara, H. Misawa, T. Nagai, and Y. Kasahara, (2003), Rebuilding process of the outer radiation belt during the 3 November 1993 magnetic storm: NOAA and Exos-D observations, $J$. Geophys. Res., 108(A1), 1004, doi:10.1029/2001JA007542.

Morley, S. K. et al. (2010), Dropouts of the outer electron radiation belt in response to solar wind stream interfaces: Global positioning system observations, Proc. Roy. Soc. A, 466, 3329-3350.

Ni, B., Y. Shprits, M. Hartinger, V. Angelopoulos, X. Gu, and D. Larson (2011), Analysis of radiation belt energetic electron phase space density using THEMIS SST measurements: Cross-satellite calibration and a case study, J. Geophys. Res., 116, A03208, doi:10.1029/ 2010JA016104.

O'Brien, T. P. and M. B. Moldwin (2003), Empirical plasmapause models from magnetic indices, Geophys. Res. Lett., 30(4), 1152, doi:10.1029/ 2002 GL016007.

O'Brien, T. P., M. D. Looper, and J. B. Blake (2004), Quantification of relativistic electron microburst losses during the GEM storms, Geophys. Res. Lett., 31, L04802, doi:10.1029/2003GL018621.

Pembroke, A., F. Toffoletto, S. Sazykin, M. Wiltberger, J. Lyon, V. Merkin, and P. Schmitt (2012), Initial results from a dynamic coupled magnetosphere-ionosphere-ring current model, J. Geophys. Res., 117, A02211, doi:10.1029/2011JA016979.

Reeves, G. D., D. N. Baker, R. D. Belian, J. B. Blake, T. E. Cayton, J. F. Fennell, R. H. W. Friedel, M. M. Meier, R. S. Selesnick, and H. E. Spence (1998), The global response of relativistic radiation belt electrons to the January 1997 magnetic cloud, Geophys. Res. Lett, 25(17), 3265-3268, doi:10.1029/98GL02509.

Reeves, G. D., K. L. McAdams, R. H. W. Friedel, and T. P. O’Brien (2003), Acceleration and loss of relativistic electrons during geomagnetic storms, Geophys. Res. Lett., 30(10), 1529, doi:10.1029/2002GL016513.

Roederer, J. G., Dynamics of Geomagnetically Trapped Radiation, p. 166, Springer-Verlag, New York, 1970.

Roux, A., O. Le Contel, C. Coillot, A. Bouabdellah, B. de la Porte, D. Alison, S. Ruocco, and M. C. Vassal (2008), The search coil magnetometer for THEMIS, Space Science Reviews, 141, 265-275, doi:10.1007/s11214008-9455-8.

Runov, A., V. Angelopoulos, and X.-Z. Zhou (2012), Multipoint observations of dipolarization front formation by magnetotail reconnection, $J$. Geophys. Res., 117, A05230, doi:10.1029/2011JA017361.

Schiller, Q., X. Li, J. Koller, H. Godinez, and D. L. Turner (2012), A parametric study of the source rate for outer radiation belt electrons using a Kalman filter, J. Geophys. Res., 117, A09211, doi:10.1029/2012JA017779.

Schulz, M., and L. J. Lanzerotti (1974), Particle diffusion in the radiation belts, Physics and Chemistry in Space, vol. 7, 215 pp., Springer-Verlag, New York.

Selesnick, R. S., and J. B. Blake (2002), Relativistic electron drift shell splitting, J. Geophys. Res., 107, 1265, doi:10.1029/2001JA009179.
Selesnick, R. S. (2006), Source and loss rates of radiation belt relativistic electrons during magnetic storms, J. Geophys. Res., 111, A04210, doi:10.1029/2005JA011473.

Shabansky, V. P. (1971), Some processes in magnetosphere, Space Sci. Rev., 12(3), 299.

Shprits, Y. Y., W. Li, and R. M. Thorne (2006a), Controlling effect of the pitch angle scattering rates near the edge of the loss cone on electron lifetimes, J. Geophys. Res., 111, A12206, doi:10.1029/2006JA011758.

Shprits, Y. Y., R. M. Thorne, R. Friedel, G. D. Reeves, J. Fennell, D. N. Baker, and S. G. Kanekal (2006b), Outward radial diffusion driven by losses at magnetopause, J. Geophys. Res., 111, A11214, doi:10.1029/ 2006JA011657.

Shprits, Y. Y., D. Kondrashov, Y. Chen, R. Thorne, M. Ghil, R. Friedel, and G. Reeves (2007), Reanalysis of relativistic radiation belt electron fluxes using CRRES satellite data, a radial diffusion model, and a Kalman filter, J. Geophys. Res., 112, A12216, doi:10.1029/2007JA012579.

Shprits, Y. Y., Elkington, S., Meredith, N. P., Subbotin, D. A. (2008a), Review of modeling of losses and sources of relativistic electrons in the outer radiation belt I: Radial transport, J. Atmos. Solar Terr. Phys., 70, doi:10.1016/j.jastp.2008.06.008.

Shprits, Y. Y., Subbotin, D. A., Meredith, N. P., Elkington, S. (2008b), Review of modeling of losses and sources of relativistic electrons in the outer radiation belt II: Local acceleration and loss, J. Atmos. Solar Terr. Phys., 70, doi:10.1016/j.jastp.2008.06.014.

Shprits, Y. Y., L. Chen, and R. M. Thorne (2009a), Simulations of pitch angle scattering of relativistic electrons with MLT dependent diffusion coefficients, J. Geophys. Res., 114, A03219, doi:10.1029/2008JA013695.

Shprits, Y. Y., D. Subbotin, and B. Ni (2009b), Evolution of electron fluxes in the outer radiation belt computed with the VERB code, J. Geophys. Res., 114, A11209, doi:10.1029/2008JA013784.

Shprits, Y. Y., M. Daae, and B. Ni (2012), Statistical analysis of phase space density buildups and dropouts, J. Geophys. Res., 117, A01219, doi:10.1029/2011JA016939.

Singer, H. J., L. Matheson, R. Grubb, A. Newman, and S. D. Bouwer (1996), Monitoring space weather with the GOES magnetometers, SPIE Conference Proceedings, 2812, GOES-8 and Beyond, edited by Edward R. Washwell, 299-308.

Summers, D., et al. (1998), Relativistic theory of wave-particle resonant diffusion with application to electron acceleration in the magnetosphere, J. Geophys. Res., 103, 20487-20500.

Summers, D., and R. M. Thorne (2003), Relativistic electron pitch-angle scattering by electromagnetic ion cyclotron waves during geomagnetic storms, J. Geophys. Res., 108, 1143, doi:10.1029/2002JA009489.

Summers, D., B. Ni, and N. P. Meredith (2007), Timescales for radiation belt electron acceleration and loss due to resonant wave-particle interactions: 2 . Evaluation for VLF chorus, ELF hiss, and electromagnetic ion cyclotron waves, J. Geophys. Res., 112, A04207, doi:10.1029/2006JA011993.

Thorne, R. M., E. J. Smith, R. K. Burton, and R. E. Holzer (1973), Plasmaspheric hiss, J. Geophys. Res., 78(10), 1581-1596, doi:10.1029/ JA078i010p01581.

Thorne, R. M., T. P. O’Brien, Y. Y. Shprits, D. Summers, and R. B. Horne (2005), Timescale for MeV electron microburst loss during geomagnetic storms, J. Geophys. Res., 110, doi:10.1029/2004JA010882.

Tsyganenko, N. A., and M. I. Sitnov (2005), Modeling the dynamics of the inner magnetosphere during strong geomagnetic storms. J. Geophys. Res., 110, A03208.

Tu, W., R. Selesnick, X. Li, and M. Looper (2010), Quantification of the precipitation loss of radiation belt electrons observed by SAMPEX, J. Geophys. Res., 115, A07210, doi:10.1029/2009JA014949.

Tu, W., and X. Li (2011), Adiabatic effects on radiation belt electrons at low altitude, J. Geophys. Res., 116, A09201, doi:10.1029/2011JA016468.

Turner D. L., and X. Li (2008), Radial gradients of phase space density of the outer radiation belt electrons prior to sudden solar wind pressure enhancements, Geophys. Res. Lett., 35, L18101.

Turner, D. L., X. Li, G. D. Reeves, and H. J. Singer (2010), On phase space density radial gradients of Earth's outer-belt electrons prior to sudden solar wind pressure enhancements: Results from distinctive events and a superposed epoch analysis, J. Geophys. Res., 115 , A01205, doi:10.1029/2009JA014423.

Turner, D. L., Y. Y. Shprits, M. Hartinger, and V. Angelopoulos (2012a), Explaining sudden losses of relativistic electrons during geomagnetic storms, Nature Phys., 8, doi:10.1038/NPHYS2185.

Turner, D. L., V. Angelopoulos, Y. Shprits, A. Kellerman, P. Cruce, and D. Larson (2012b), Radial distributions of equatorial phase space density for outer radiation belt electrons, Geophys. Res. Lett., 39, L09101, doi:10.1029/2012GL051722.

Turner, D. L., S. K. Morley, Y. Miyoshi, B. Ni, and C.-L. Huang (2012c), Outer radiation belt flux dropouts: Current understanding and unresolved questions, Dynamics of Earth's Radiation Belts and Inner Magnetosphere, Geophysical Monograph Series, Vol. 199, Edited by D. Summers, 


\section{TURNER ET AL.: STORM-TIME EVOLUTION OF THE OUTER BELT}

I. R. Mann, D. N. Baker, and M. Schulz, pp. 195-212, AGU, Washington, D.C., doi:10.1029/2012GM001310.

Ukhorskiy, A. Y., B. J. Anderson, P. C. Brandt, and N. A. Tsyganenko (2006), Storm time evolution of the outer radiation belt: Transport and losses, J. Geophys. Res., 111, A11S03, doi:10.1029/2006JA011690.

Ukhorskiy, A. Y., Y. Y. Shprits, B. J. Anderson, K. Takahashi, and R. M. Thorne (2010), Rapid scattering of radiation belt electrons by storm-time EMIC waves, Geophys. Res. Lett., 37, L09101, doi:10.1029/2010GL042906.

Ukhorskiy, A. Y., M. I. Sitnov, R. M. Millan, and B. T. Kress (2011), The role of drift orbit bifurcations in energization and loss of electrons in the outer radiation belt, J. Geophys. Res., 116, A09208, doi:10.1029/2011JA016623.

Usanova, M. E., I. R. Mann, I. J. Rae, Z. C. Kale, V. Angelopoulos, J. W. Bonnell, K.-H. Glassmeier, H. U. Auster, and H. J. Singer (2008),
Multipoint observations of magnetospheric compression-related EMIC Pc1 waves by THEMIS and CARISMA, Geophys. Res. Lett., 35, L17S25, doi:10.1029/2008GL034458

Usanova, M. E., I. R. Mann, J. Bortnik, L. Shao, V. Angelopoulos (2012), THEMIS observations of EMIC wave occurrence: Dependence on AE, SYMH, and solar wind dynamic pressure, J. Geophys. Res., 117, A10218, doi:10.1029/2012JA018049.

Yando, K., R. M. Millan, J. C. Green, and D. S. Evans (2011), A Monte Carlo simulation of the NOAA POES Medium Energy Proton and Electron Detector instrument, J. Geophys. Res., 116, A10231, doi:10.1029/2011JA016671.

Zhao, H., and X. Li (2013), Inward shift of outer radiation belt electrons as a function of Dst index and the influence of the solar wind on electron injections into the slot region, J. Geophys. Res., doi:10.1029/2012JA018179. 